\title{
Graph homomorphisms between trees
}

\author{
Péter Csikvári \\ Department of Mathematics, Massachusetts Institute of Technology \\ Cambridge, MA 02139 \\ Department of Computer Science, Eötvös Loránd University \\ H-1117 Budapest, Pázmány Péter sétány 1/C, Hungary \\ peter.csikvari@gmail.com \\ Zhicong Lin \\ Department of Mathematics and Statistics, Lanzhou University \\ Lanzhou 730000, P.R. China \\ Institut Camille Jordan, Université Claude Bernard Lyon 1 \\ 43 boulevard du 11 novembre 1918, F-69622 Villeurbanne, France \\ lin@math.univ-lyon1.fr
}

Submitted: Feb 8, 2014; Accepted: Sep 26, 2014; Published: Oct 9, 2014

Mathematics Subject Classifications: 05C05, 05C30, 05C35

\begin{abstract}
In this paper we study several problems concerning the number of homomorphisms of trees. We begin with an algorithm for the number of homomorphisms from a tree to any graph. By using this algorithm and some transformations on trees, we study various extremal problems about the number of homomorphisms of trees. These applications include a far reaching generalization and a dual of Bollobás and Tyomkyn's result concerning the number of walks in trees.

Some other main results of the paper are the following. Denote by $\operatorname{hom}(H, G)$ the number of homomorphisms from a graph $H$ to a graph $G$. For any tree $T_{m}$ on $m$ vertices we give a general lower bound for $\operatorname{hom}\left(T_{m}, G\right)$ by certain entropies of Markov chains defined on the graph $G$. As a particular case, we show that for any graph $G$,

$$
\exp \left(H_{\lambda}(G)\right) \lambda^{m-1} \leqslant \operatorname{hom}\left(T_{m}, G\right),
$$

where $\lambda$ is the largest eigenvalue of the adjacency matrix of $G$ and $H_{\lambda}(G)$ is a certain constant depending only on $G$ which we call the spectral entropy of $G$. We also show that if $T_{m}$ is any fixed tree and

$$
\operatorname{hom}\left(T_{m}, P_{n}\right)>\operatorname{hom}\left(T_{m}, T_{n}\right),
$$

for some tree $T_{n}$ on $n$ vertices, then $T_{n}$ must be the tree obtained from a path $P_{n-1}$ by attaching a pendant vertex to the second vertex of $P_{n-1}$.
\end{abstract}


All the results together enable us to show that among all trees with fixed number of vertices, the path graph has the fewest number of endomorphisms while the star graph has the most.

Keywords: trees; walks; graph homomorphisms; adjacency matrix; extremal problems; KC-transformation; Markov chains

\section{Introduction}

We use standard notations and terminology of graph theory, see for instance $[2,4]$. The graphs considered here are finite and undirected without multiple edges and loops. Given a graph $G$, we write $V(G)$ for the vertex set and $E(G)$ for the edge set. A homomorphism from a graph $H$ to a graph $G$ is a mapping $f: V(H) \rightarrow V(G)$ such that the images of adjacent vertices are adjacent. Let $\operatorname{Hom}(H, G)$ denote the set of homomorphisms from $H$ to $G$ and by $\operatorname{hom}(H, G)$ the number of homomorphisms from $H$ to $G$. Throughout this article, we write $P_{n}$ and $S_{n}$ for the path and the star on $n$ vertices, respectively. The length of a path is the number of its edges. The union of graphs $G$ and $H$ is the graph $G \cup H$ with vertex set $V(G) \cup V(H)$ and edge set $E(G) \cup E(H)$. A tree $T$ together with a root vertex $v$ will be denoted by $T(v)$.

The problem of computing $\operatorname{hom}(H, G)$ is difficult in general. However, there has been recent interest in counting homomorphisms between special graphs. In particular, formulas for computing the number of homomorphisms between two different paths were given in $[1,16]$. But even for these special trees, the formulas are bulky and inelegant. In Section 2, we shall give an algorithm for computing the number of homomorphisms from trees to any graph. This algorithm will be called Tree-walk algorithm.

Recently, the first author proved a conjecture of Nikiforov concerning the number of closed walks on trees. He proved in [6] that, for a fixed integer $m$, the number of closed walks of length $m$ on trees of order $n$ attains its maximum at the star $S_{n}$ and its minimum at the path $P_{n}$. In other words,

$$
\operatorname{hom}\left(C_{m}, P_{n}\right) \leqslant \operatorname{hom}\left(C_{m}, T_{n}\right) \leqslant \operatorname{hom}\left(C_{m}, S_{n}\right)
$$

where $T_{n}$ is a tree on $n$ vertices and $C_{m}$ is the cycle on $m$ vertices.

Bollobás and Tyomkyn [3] gave a variant of the first author's result by replacing the number of closed walks by the number of all walks, that is

$$
\operatorname{hom}\left(P_{m}, P_{n}\right) \leqslant \operatorname{hom}\left(P_{m}, T_{n}\right) \leqslant \operatorname{hom}\left(P_{m}, S_{n}\right)
$$

where $T_{n}$ is a tree on $n$ vertices. In both [3] and [6], the authors use a certain transformation of trees. In [6], it is called the generalized tree shift, whereas in [3], it is renamed to $K C$-transformation.

To define this transformation, let $x$ and $y$ be two vertices of a tree $T$ such that every interior vertex of the unique $x-y$ path $P$ in $T$ has degree two, and write $z$ for the neighbor of $y$ on this path. Let $N(v)$ denote the set of neighbors of a vertex $v$. The 

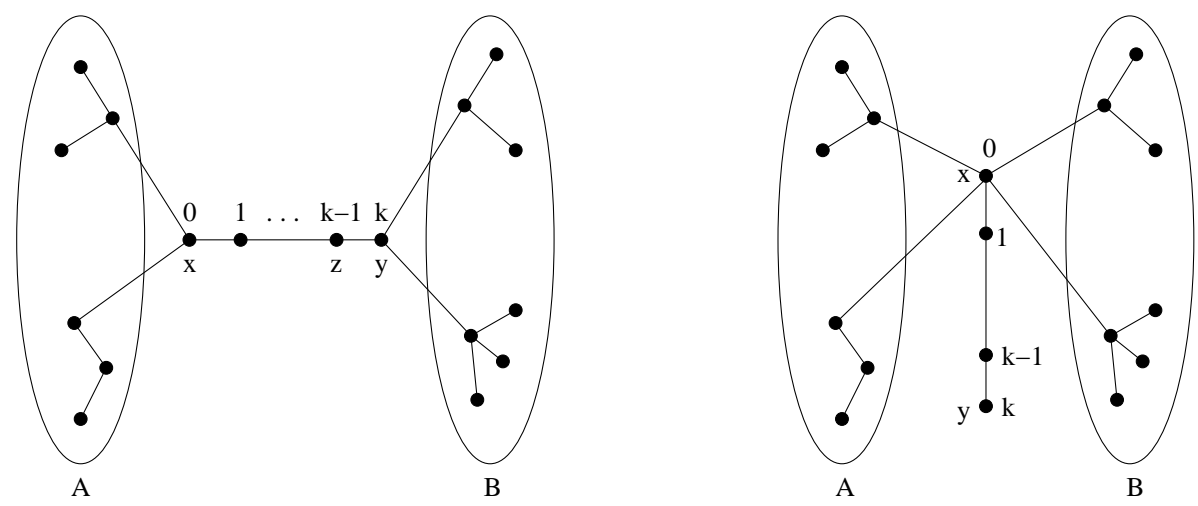

Figure 1: The KC-transformation.

KC-transformation, $K C(T, x, y)$, of the tree $T$ with respect to the path $P$ is obtained from $T$ by deleting all edges between $y$ and $N(y) \backslash z$ and adding the edges between $x$ and $N(y) \backslash z$ instead (See Fig. 1). Note that $K C(T, x, y)$ and $K C(T, y, x)$ are isomorphic.

The following property of KC-transformation was proved in [6].

Proposition 1.1. The $K C$-transformation gives rise to a graded poset of trees on $n$ vertices (graded by the number of leaves) with the star as the largest and the path as the smallest element. See Figure 2.

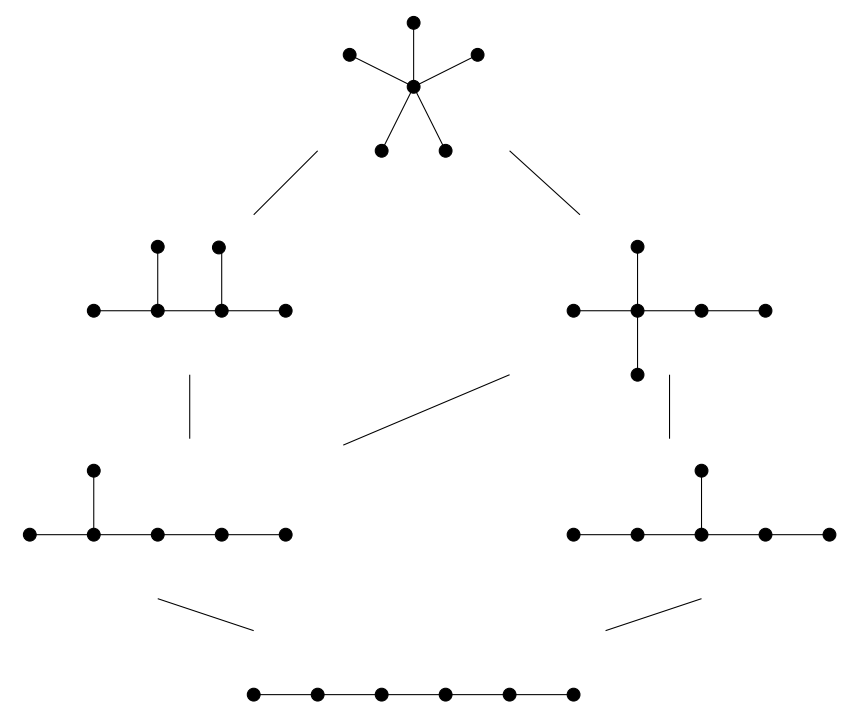

Figure 2: The induced poset of KC-transformation on trees of 6 vertices.

In [6] the first author proved that the KC-transformation increases the number of closed walks of fixed length in trees. By Proposition 1.1, this leads to the proof of inequality (1.1). 
In the very same spirit, Bollobás and Tyomkyn [3] showed that the KC-transformation increases the number of walks of fixed length in trees. In the language of graph homomorphism, their result can be restated as follows.

Theorem 1.2 (Bollobás-Tyomkyn). Let $T$ be a tree and let $T^{\prime}$ be obtained from $T$ by a KC-transformation. Then

$$
\operatorname{hom}\left(P_{m}, T^{\prime}\right) \geqslant \operatorname{hom}\left(P_{m}, T\right)
$$

for any $m \geqslant 1$.

Now a natural question arises: does inequality (1.3) still hold when $P_{m}$ is replaced by an arbitrary fixed tree? A tree is called starlike if it has at most one vertex of degree greater than two. Note that paths are starlike. We answer this question in the affirmative for starlike trees.

Theorem 1.3. Let $T$ be a tree and $T^{\prime}$ the $K C$-transformation of $T$ with respect to a path of length $k$. Then the inequality

$$
\operatorname{hom}\left(H, T^{\prime}\right) \geqslant \operatorname{hom}(H, T)
$$

holds when $k$ is even and $H$ is any tree, or $k$ is odd and $H$ is a starlike tree.

Moreover, we find a counterexample for inequality (1.4) when $k$ is odd and $H$ is not a starlike tree (see the end of Section 3).

Another extremal problem concerning the number of homomorphisms between trees that worth considering is to find the extremal trees for hom $\left(\cdot, P_{n}\right)$ over all trees on $m$ vertices. We address this question in a follow-up paper [8], here we only mention the main result of this paper. This result can be considered as a dual of inequality (1.2).

Theorem 1.4. Let $T_{m}$ be a tree on $m$ vertices. Furthermore, let $\operatorname{diam}\left(T_{m}\right)$ denote the diameter of $T_{m}$.

(i) Let $T_{m}^{\prime}$ be obtained from $T_{m}$ by a KC-transformation. If $n$ is even, or $n$ is odd and $\operatorname{diam}\left(T_{m}\right) \leqslant n-1$, then

$$
\operatorname{hom}\left(T_{m}, P_{n}\right) \leqslant \operatorname{hom}\left(T_{m}^{\prime}, P_{n}\right)
$$

(ii) For any $m, n$,

$$
\operatorname{hom}\left(P_{m}, P_{n}\right) \leqslant \operatorname{hom}\left(T_{m}, P_{n}\right) \leqslant \operatorname{hom}\left(S_{m}, P_{n}\right) .
$$

As we mentioned, the proof of Theorem 1.4 will be given in [8], it only builds on the algorithm of Section 2. Note that inequality (1.5) is not true in general when $n$ is odd and $\operatorname{diam}\left(T_{m}\right)$ is greater than $n-1$.

For the sake of keeping this paper self-contained, we will also give a new proof for the following theorem of Sidorenko [19] concerning the extremal property of the stars among trees. Note that Fiol and Garriga [10] proved the special case of this theorem when $T_{m}=P_{m}$, clearly, they were not aware of the work of Sidorenko. 
Theorem 1.5 (Sidorenko). Let $G$ be an arbitrary graph and let $T_{m}$ be a tree on $m$ vertices. Then

$$
\operatorname{hom}\left(T_{m}, G\right) \leqslant \operatorname{hom}\left(S_{m}, G\right)
$$

After all, it is a natural question whether it is true or not that

$$
\operatorname{hom}\left(P_{m}, G\right) \leqslant \operatorname{hom}\left(T_{m}, G\right)
$$

for any tree $T_{m}$ on $m$ vertices. Surprisingly, the answer is no! It was already known to A. Leontovich [14]. It turns out that even if one restricts $G$ to be a tree there is a counterexample (see Remark 4.13).

We have already seen a few examples to the phenomenon that in many extremal problems concerning trees it turns out that the maximal (minimal) value of the examined parameter is attained at the star and the minimal (maximal) value is attained at the path among trees on $n$ vertices (cf. $[7,17]$ ). In what follows we will show that this phenomenon occurs quite frequently if one studies homomorphisms of trees.

Let $Y_{a, b, c}$ be the starlike tree on $a+b+c+1$ vertices which has exactly 3 leaves and the vertex of degree 3 has distance $a, b, c$ from the leaves, respectively.

Theorem 1.6. Let $T_{n}$ be a tree on $n$ vertices. Assume that for a tree $T_{m}$ we have

$$
\operatorname{hom}\left(T_{m}, T_{n}\right)<\operatorname{hom}\left(T_{m}, P_{n}\right)
$$

Then $T_{n}=Y_{1,1, n-3}$ and $n$ is even.

In fact, we conjecture that we only have to exclude the case $n=4$ and $T_{4}=S_{4}$.

Conjecture 1.7. Let $T_{n}$ be a tree on $n$ vertices, where $n \geqslant 5$. Then for any tree $T_{m}$ we have

$$
\operatorname{hom}\left(T_{m}, P_{n}\right) \leqslant \operatorname{hom}\left(T_{m}, T_{n}\right) \text {. }
$$

An endomorphism of a graph is a homomorphism from the graph to itself. For a graph $G$, denote by $\operatorname{End}(G)$ the set of endomorphisms of $G$. We remark that $\operatorname{End}(G)$ forms a monoid with respect to the composition of mappings. One of the main results of this paper is the following extremal property about the number of endomorphisms of trees.

Theorem 1.8. For all trees $T_{n}$ on $n$ vertices we have

$$
\left|\operatorname{End}\left(P_{n}\right)\right| \leqslant\left|\operatorname{End}\left(T_{n}\right)\right| \leqslant\left|\operatorname{End}\left(S_{n}\right)\right| .
$$

Both the proofs of Theorem 1.6 and the first part of Theorem 1.8 require a crucial lower bound involving Markov chains for the number of graph homomorphisms from trees (see Theorem 4.1). Our lower bound generalizes a recent result due to Dellamonica et al. [9] (by choosing the classical Markov chain on graphs) and is also closely related to works of Kopparty and Rossman [13] and Rossman and Vee [21]. The idea of studying homomorphisms via entropies of Markov chains on graphs seems new. 
The rest of this paper is organized as follows. In Section 2, we state the tree-walk algorithm. Section 3 is devoted to the proof of Theorem 1.3. In Section 4, we prove some lower bounds involving Markov chains and an upper bound (Theorem 1.5) for the number of homomorphisms from trees to an arbitrary graph. The proofs of Theorem 1.6 and Theorem 1.8 are given in Section 5, where some lower bounds concerning the homomorphisms of arbitrary trees are also proved.

In order to make our paper transparent, we offer the following two tables, Figure 3 and 4, which summarize our results. In both tables, the first row follows from Theorem 1.2 or its generalization Corollary 3.3. The last row is obvious since $\operatorname{hom}\left(S_{m}, G\right)$ is the sum of degree powers of $G$ and it also follows from Corollary 3.3. The first, second and third columns follow from Theorem 1.4, Theorem 1.5 and Corollary 3.5 respectively. The " $X$ " means that there is no inequality between the two expressions in general and the "?" means that we do not know whether the statement is true or not.

$$
\begin{aligned}
& \operatorname{hom}\left(P_{m}, P_{n}\right) \leqslant \operatorname{hom}\left(P_{m}, T_{n}\right) \leqslant \operatorname{hom}\left(P_{m}, S_{n}\right) \\
& \wedge \quad X \wedge \\
& \operatorname{hom}\left(T_{m}, P_{n}\right) \stackrel{(*)}{\leqslant} \operatorname{hom}\left(T_{m}, T_{n}\right) \quad X \operatorname{hom}\left(T_{m}, S_{n}\right) \\
& \wedge \wedge \wedge \wedge \\
& \operatorname{hom}\left(S_{m}, P_{n}\right) \leqslant \operatorname{hom}\left(S_{m}, T_{n}\right) \leqslant \operatorname{hom}\left(S_{m}, S_{n}\right)
\end{aligned}
$$

Figure 3: The number of homomorphisms between trees of sizes $m$ and $n$. The $(*)$ means that there are some well-determined (possible) counterexamples which should be excluded.

$$
\begin{array}{ccc}
\operatorname{hom}\left(P_{n}, P_{n}\right) & \leqslant \operatorname{hom}\left(P_{n}, T_{n}\right) \leqslant \operatorname{hom}\left(P_{n}, S_{n}\right) \\
\wedge & ? & \wedge \\
\operatorname{hom}\left(T_{n}, P_{n}\right) & \leqslant \operatorname{hom}\left(T_{n}, T_{n}\right) & X \\
\wedge & \operatorname{hom}\left(T_{n}, S_{n}\right) \\
\operatorname{hom}\left(S_{n}, P_{n}\right) & \leqslant \operatorname{hom}\left(S_{n}, T_{n}\right) \leqslant \operatorname{hom}\left(S_{n}, S_{n}\right)
\end{array}
$$

Figure 4: The number of homomorphisms and endomorphisms of trees of size $n$.

\section{The Tree-walk algorithm}

In this section we shall state an algorithm for the number of homomorphisms from a tree to any graph. As a generalized concept of walks in graphs, we call a homomorphism from a tree to a graph a tree-walk on this graph. 
Let $\mathbf{a}=\left(a_{1}, a_{2}, \ldots, a_{n}\right)$ and $\mathbf{b}=\left(b_{1}, b_{2}, \ldots, b_{n}\right)$ be two vectors. We usually denote by $\|\mathbf{a}\|=a_{1}+a_{2}+\cdots+a_{n}$ the norm of $\mathbf{a}$ and by $\mathbf{a} * \mathbf{b}=\left(a_{1} b_{1}, \ldots, a_{n} b_{n}\right)$ the Hadamard product of $\mathbf{a}$ and $\mathbf{b}$. Denote by $\mathbf{1}_{\mathbf{n}}$ the $n$-dimensional row vector with all entries are equal to 1 . Let $G$ be a graph with $n$ vertices. The adjacency matrix of $G$ is the $n \times n$ matrix $A_{G}:=\left(a_{u v}\right)_{u, v \in V(G)}$, where $a_{u v}=1$ when $u v \in E(G)$, otherwise 0 . We begin with a fundamental lemma about the number of walks in a graph.

Lemma 2.1. Let $G$ be a labeled graph and $A=A_{G}$ the adjacency matrix of $G$. Then the $(i, j)$-entry of the matrix $A^{n}$ counts the number of walks in $G$ from vertex $i$ to vertex $j$ with length $n$.

Proof. By easy induction on $n$. See for example [20, Theorem 4.7.1].

Definition 2.2 (hom-vector). Let $T$ be a tree and $G$ be a graph with vertices labeled by $1,2, \ldots, n$. Let $v \in V(T)$ be any vertex of $T$. The $n$-dimensional vector

$$
\mathbf{h}(T, v, G):=\left(h_{1}, h_{2}, \ldots, h_{n}\right)
$$

where

$$
h_{i}=|\{f \in \operatorname{Hom}(T, G) \mid f(v)=i\}|,
$$

is called the hom-vector at $v$ from $T$ to $G$. Clearly, $\operatorname{hom}(T, G)=\|\mathbf{h}(T, v, G)\|$. Sometimes, we also call $\mathbf{h}(T, v, G)$ the hom-vector from the rooted tree $T(v)$ to the graph $G$ and use the more compact notation $\mathbf{h}(T(v), G)$.

The following Tree-walk algorithm can be viewed as a generalization of Lemma 2.1 for computing the number of tree-walks in graphs.

The Tree-walk algorithm. Let $A=A_{G}$ be the adjacency matrix of the labeled graph $G$. Let $v$ be a vertex of the tree $T$. We now give the algorithm to compute $\mathbf{h}(T, v, G)$. We consider two type of recursion steps.

Recursion 1. If $v$ is a non-leaf vertex of $T$, then we can decompose $T$ to $T_{1} \cup T_{2}$ such that $V\left(T_{1}\right) \cap V\left(T_{2}\right)=\{v\}$, and $T_{1}$ and $T_{2}$ are strictly smaller than $T$. In this case

$$
\mathbf{h}(T, v, G)=\mathbf{h}\left(T_{1}, v, G\right) * \mathbf{h}\left(T_{2}, v, G\right) .
$$

Recursion 2. If $v$ is a leaf with the unique neighbor $u$ in $T$, then

$$
\mathbf{h}(T, v, G)=\mathbf{h}(T-v, u, G) A .
$$

Hence we use Recursion 1 or Recursion 2 according to the vertex $v$ is a non-leaf or a leaf. In most of the proofs we simply check whether some property of the vector $\mathbf{h}(T, v, G)$ remains valid after applying Recursion 1 and Recursion 2.

Note that if a leaf has distance $d$ from the closest vertex of degree at least 3 then we can execute a sequence of Recursion 2 in one step by simply multiplying the corresponding hom-vector by $A^{d}$. This way we can speed up the algorithm a bit. For the sake of convenience, we include an example. 

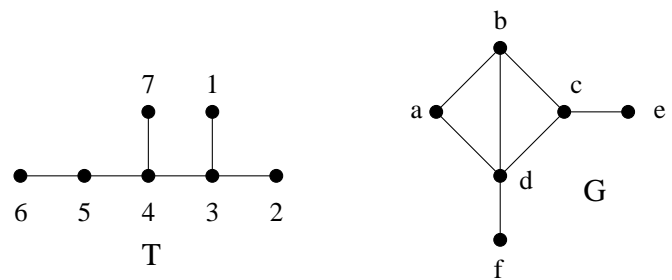

Figure 5: A labeled tree $T$ and a graph $G$.

Example 2.3. Let $T$ and $G$ be the tree and the graph depicted in Fig. 5. Denote by $T[V]$ the induced subtree on the vertex set $V \subseteq V(T)$. Let us compute $\mathbf{h}(T, 7, G)$ by the Tree-walk algorithm. First, we compute $\mathbf{h}(T[1,3], 3, G)$ by using Recursion 2:

$$
\mathbf{h}(T[1,3], 3, G)=\mathbf{1}_{\mathbf{7}} A_{G}=(2,3,3,4,1,1) .
$$

Next we compute $\mathbf{h}(T[1,2,3], 3, G)$ by using Recursion 1 :

$$
\mathbf{h}(T[1,2,3], 3, G)=\mathbf{h}(T[1,3], 3, G) * \mathbf{h}(T[2,3], 3, G)=(4,9,9,16,1,1) .
$$

Now let us compute $\mathbf{h}(T[1,2,3,4], 4, G)$ by using Recursion 2 again:

$$
\mathbf{h}(T[1,2,3,4], 4, G)=\mathbf{h}(T[1,2,3], 3, G) A_{G}=(25,29,26,23,9,16) .
$$

As a next step we determine $\mathbf{h}(T[6,5,4], 4, G)$ :

$$
\mathbf{h}(T[6,5,4], 4, G)=\mathbf{1}_{\mathbf{7}} A_{G}^{2}=(7,9,8,9,3,4) .
$$

Hence

$$
\begin{aligned}
\mathbf{h}(T[1,2,3,4,5,6], 4, G) & =\mathbf{h}(T[1,2,3,4], 4, G) * \mathbf{h}(T[6,5,4], 4, G) \\
& =(175,261,208,207,27,64) .
\end{aligned}
$$

Finally,

$$
\mathbf{h}(T, 7, G)=\mathbf{h}(T[1,2,3,4,5,6], 4, G) A_{G}=(468,590,495,708,208,207) .
$$

Thus $\operatorname{hom}(T, G)=\|\mathbf{h}(T, 7, G)\|=2676$.

\section{Proof of Theorem 1.3}

The main purpose of this section is to prove Theorem 1.3. We shall give an inductive proof of Theorem 1.2 which can be generalized to tree-walks by the tree-walk algorithm.

We first need some notations. Let $T$ be a tree and $T^{\prime}=K C\left(T, p_{0}, p_{k}\right)$ its $\mathrm{KC}$ transformation with respect to a path $P$ of length $k$, a path with vertices labeled consecutively with $p_{0}, p_{1}, \ldots, p_{k}$. We denote by $A$ and $B$ the components of $p_{0}$ and $p_{k}$ in the 
subgraph of $T$ by deleting all the edges of $P$. Let $A^{\prime}, B^{\prime}$ and $P^{\prime}$ be the components of $T^{\prime}$ corresponding with components $A, B$ and $P$ under the KC-transformation, respectively. The vertices of the path $P^{\prime}$ will be labeled consecutively with $p_{0}^{\prime}, p_{1}^{\prime}, \ldots, p_{k}^{\prime}$, where $p_{i}^{\prime}$ is corresponding to $p_{i}$ for $0 \leqslant i \leqslant k$. So $p_{0} \in A, p_{k} \in B$ in $T$, and $p_{0}^{\prime} \in A^{\prime}, B^{\prime}$ in $T^{\prime}$.

Lemma 3.1. Let $a_{1}, a_{2}, b_{1}, b_{2}, c_{1}, c_{2}, d_{1}, d_{2}$ be positive numbers satisfying the inequalities: $a_{i} \geqslant \max \left(c_{i}, d_{i}\right), a_{i}+b_{i} \geqslant c_{i}+d_{i}$ for $i=1,2$. Then $a_{1} a_{2} \geqslant \max \left(c_{1} c_{2}, d_{1} d_{2}\right)$ and $a_{1} a_{2}+$ $b_{1} b_{2} \geqslant c_{1} c_{2}+d_{1} d_{2}$.

Proof. Clearly, we only have to prove that $a_{1} a_{2}+b_{1} b_{2} \geqslant c_{1} c_{2}+d_{1} d_{2}$, the other inequality is trivial. Note that $b_{i} \geqslant \max \left(0, c_{i}+d_{i}-a_{i}\right)$. If one of $c_{i}+d_{i}-a_{i}<0$, say $c_{1}+d_{1}<a_{1}$ then

$$
a_{1} a_{2} \geqslant\left(c_{1}+d_{1}\right) a_{2} \geqslant c_{1} c_{2}+d_{1} d_{2} .
$$

If both $c_{i}+d_{i}-a_{i} \geqslant 0$ for $i=1,2$, then

$$
\begin{aligned}
& a_{1} a_{2}+b_{1} b_{2} \geqslant a_{1} a_{2}+\left(c_{1}+d_{1}-a_{1}\right)\left(c_{2}+d_{2}-a_{2}\right) \\
= & c_{1} c_{2}+d_{1} d_{2}+\left(a_{1}-c_{1}\right)\left(a_{2}-d_{2}\right)+\left(a_{1}-d_{1}\right)\left(a_{2}-c_{2}\right) \geqslant c_{1} c_{2}+d_{1} d_{2} .
\end{aligned}
$$

Hence we are done.

We first treat the case of $k$ being even.

Proof of first part of Theorem 1.3. In this proof $k$ is even: $k=2 t$. We label $V(A) \backslash p_{0}$ with $\left\{a_{m} \mid 1 \leqslant m \leqslant M\right\}, V\left(A^{\prime}\right) \backslash p_{0}^{\prime}$ with $\left\{a_{i}^{\prime} \mid 1 \leqslant m \leqslant M\right\}, V(B) \backslash p_{k}$ with $\left\{b_{n} \mid 1 \leqslant n \leqslant N\right\}$ and $V(B) \backslash p_{0}^{\prime}$ with $\left\{b_{n}^{\prime} \mid 1 \leqslant n \leqslant N\right\}$, where $a_{m}$ (resp. $b_{n}$ ) is corresponding to $a_{m}^{\prime}$ (resp. $\left.b_{n}^{\prime}\right)$ under the KC-transformation. For $v \in H$, we always write

$$
\begin{aligned}
\mathbf{h}(H, v, T) & =\left(a_{1}, a_{2}, \ldots, a_{M}, p_{0}, p_{1}, \ldots, p_{k}, b_{1}, b_{2}, \ldots, b_{N}\right) \text { and } \\
\mathbf{h}\left(H, v, T^{\prime}\right) & =\left(a_{1}^{\prime}, a_{2}^{\prime}, \ldots, a_{M}^{\prime}, p_{0}^{\prime}, p_{1}^{\prime}, \ldots, p_{k}^{\prime}, b_{1}^{\prime}, b_{2}^{\prime}, \ldots, b_{N}^{\prime}\right),
\end{aligned}
$$

where we use the labels of vertices of $T$ and $T^{\prime}$ to index the parameters of the hom-vectors to $T$ and $T^{\prime}$ respectively. We hope that it will not cause any confusion. We shall prove by induction on the steps of tree-walk algorithm that

$$
\begin{array}{r}
a_{m}^{\prime} \geqslant a_{m}, \quad b_{n}^{\prime} \geqslant b_{n} \\
p_{i}^{\prime}+p_{k-i}^{\prime} \geqslant p_{i}+p_{k-i} \\
p_{i}^{\prime} \geqslant p_{i}, p_{i}^{\prime} \geqslant p_{k-i}
\end{array}
$$

for $1 \leqslant m \leqslant M, 1 \leqslant n \leqslant N, 0 \leqslant i \leqslant t$.

It is easy to verify that all these inequalities are satisfied after applying any recursion step of the tree-walk algorithm. When $v$ is a leaf of $H$ then it is trivial that these inequalities are preserved. If $v$ is not a leaf then we use Lemma 3.1 to see that the Hadamard-product preserves these inequalities. 
Lemma 3.2. Let $k$ be odd and assume that $A$ and $B$ have at least two vertices. Let $a_{m}(r), b_{n}(r), p_{i}(r), a_{m}^{\prime}(r), b_{n}^{\prime}(r), p_{i}^{\prime}(r)$ denote the number of homomorphism of $P_{r}$ into $T$ and $T^{\prime}$, respectively, such that the endvertex of $P_{r}$ goes to the vertices $a_{m}, b_{n}, p_{i}, a_{m}^{\prime}, b_{n}^{\prime}$ and $p_{i}^{\prime}$, respectively. Then the following inequalities hold for every $r$ :

$$
\begin{array}{r}
a_{m}^{\prime}(r) \geqslant a_{m}(r), b_{n}^{\prime}(r) \geqslant b_{n}(r) \\
p_{i}^{\prime}(r)+p_{j}^{\prime}(r) \geqslant p_{i}(r)+p_{j}(r) \\
p_{i}^{\prime}(r)+p_{j}^{\prime}(r) \geqslant p_{k-i}(r)+p_{k-j}(r)
\end{array}
$$

for $1 \leqslant m \leqslant M, 1 \leqslant n \leqslant N$ and $i+j \leqslant k$.

Proof. We prove the claim by induction on $r$. For $r=1,2$, the claim is trivial. Note that we only have to prove that

$$
\begin{array}{r}
a_{m}^{\prime}(r) \geqslant a_{m}(r), b_{n}^{\prime}(r) \geqslant b_{n}(r) \\
p_{i}^{\prime}(r)+p_{j}^{\prime}(r) \geqslant p_{i}(r)+p_{j}(r)
\end{array}
$$

for $1 \leqslant m \leqslant M, 1 \leqslant n \leqslant N$ and $i+j \leqslant k$. We obtain the inequality

$$
p_{i}^{\prime}(r)+p_{j}^{\prime}(r) \geqslant p_{k-i}(r)+p_{k-j}(r)
$$

by simply exchanging the role of $A$ and $B$. Also note that if we put $i=j$ in the inequality (3.2) and (3.3) we obtain that $p_{i}^{\prime}(r) \geqslant p_{i}(r), p_{k-i}(r)$ for $i<k / 2$.

Observe that for any vertex $v$ we have:

$$
v(r)=\sum_{u \in N(v)} u(r-1)
$$

We will treat the cases $k=1$ and $k \geqslant 3$ separately.

Case 1: $\boldsymbol{k}=\mathbf{1}$. In this case, we have to prove the inequalities:

$$
a_{m}^{\prime}(r) \geqslant a_{m}(r), b_{n}^{\prime}(r) \geqslant b_{n}(r), p_{0}^{\prime}(r) \geqslant \max \left(p_{0}(r), p_{1}(r)\right), p_{0}^{\prime}(r)+p_{1}^{\prime}(r) \geqslant p_{0}(r)+p_{1}(r) .
$$

The inequalities $a_{m}^{\prime}(r) \geqslant a_{m}(r), b_{n}^{\prime}(r) \geqslant b_{n}(r)$ simply follow from the inequalities $a_{m}^{\prime}(r-1) \geqslant a_{m}(r-1), b_{n}^{\prime}(r-1) \geqslant b_{n}(r-1)$, and $p_{0}^{\prime}(r-1) \geqslant p_{0}(r-1), p_{1}(r-1)$.

Observe that

$$
\begin{aligned}
p_{0}^{\prime}(r) & =\sum_{a_{m}^{\prime} \in N\left(p_{0}^{\prime}\right)} a_{m}^{\prime}(r-1)+\sum_{b_{n}^{\prime} \in N\left(p_{0}^{\prime}\right)} b_{n}^{\prime}(r-1)+p_{1}^{\prime}(r-1) \\
& =\sum_{a_{m}^{\prime} \in N\left(p_{0}^{\prime}\right)} a_{m}^{\prime}(r-1)+\sum_{b_{n}^{\prime} \in N\left(p_{0}^{\prime}\right)} b_{n}^{\prime}(r-1)+p_{0}^{\prime}(r-2) \\
& \geqslant \sum_{a_{m} \in N\left(p_{0}\right)} a_{m}(r-1)+\sum_{b_{n} \in N\left(p_{1}\right)} b_{n}(r-1)+p_{0}(r-2) \\
& \geqslant \sum_{a_{m} \in N\left(p_{0}\right)} a_{m}(r-1)+\sum_{b_{n} \in N\left(p_{1}\right)} b_{n}(r-2)+p_{0}(r-2)
\end{aligned}
$$




$$
=\sum_{a_{m} \in N\left(p_{0}\right)} a_{m}(r-1)+p_{1}(r-1)=p_{0}(r) .
$$

We used the induction hypothesis and that $b_{m}(r-1) \geqslant b_{m}(r-2)$. In general, $u(r) \geqslant$ $u(r-1)$ since any homomorphism of $P_{r-1}$ starting at the vertex $u$ can be extended to a homomorphism of $P_{r}$ starting at $u$. Clearly, we can get $p_{0}^{\prime}(r) \geqslant p_{1}(r)$ similarly, or we just switch the role of $A$ and $B$.

Finally,

$$
\begin{aligned}
& p_{0}^{\prime}(r)+p_{1}^{\prime}(r) \\
= & \sum_{a_{m}^{\prime} \in N\left(p_{0}^{\prime}\right)} a_{m}^{\prime}(r-1)+\sum_{b_{n}^{\prime} \in N\left(p_{0}^{\prime}\right)} b_{n}^{\prime}(r-1)+p_{0}^{\prime}(r-1)+p_{1}^{\prime}(r-1) \\
\geqslant & \sum_{a_{m} \in N\left(p_{0}\right)} a_{m}(r-1)+\sum_{b_{n} \in N\left(p_{1}\right)} b_{n}(r-1)+p_{0}(r-1)+p_{1}(r-1)=p_{0}(r)+p_{1}(r) .
\end{aligned}
$$

Hence we are done in this case.

Case 2: $\boldsymbol{k} \geqslant 3$. Clearly, the inequalities $a_{m}^{\prime}(r) \geqslant a_{m}(r), b_{n}^{\prime}(r) \geqslant b_{n}(r)$ simply follow from the inequalities $a_{m}^{\prime}(r-1) \geqslant a_{m}(r-1), b_{n}^{\prime}(r-1) \geqslant b_{n}(r-1)$, and $p_{0}^{\prime}(r-1) \geqslant$ $p_{0}(r-1), p_{k}(r-1)$ as before.

So we only have to prove the inequality $p_{i}^{\prime}(r)+p_{j}^{\prime}(r) \geqslant p_{i}(r)+p_{j}(r)$ for $i+j \leqslant k$. We can assume that $i \leqslant j$. If $i \geqslant 1$, then $j \leqslant k-1$ and

$$
\begin{aligned}
p_{i}^{\prime}(r)+p_{j}^{\prime}(r) & =\left(p_{i-1}^{\prime}(r-1)+p_{j+1}^{\prime}(r-1)\right)+\left(p_{i+1}^{\prime}(r-1)+p_{j-1}^{\prime}(r-1)\right) \\
& \geqslant\left(p_{i-1}(r-1)+p_{j+1}(r-1)\right)+\left(p_{i+1}(r-1)+p_{j-1}(r-1)\right) \\
& =p_{i}(r)+p_{j}(r) .
\end{aligned}
$$

So we only have to consider the case $i=0$. In this case we consider the cases $j=$ $0, j=1,2 \leqslant j \leqslant k-2, j=k-1, j=k$ separately. Unfortunately, all of them behaves a bit differently.

Subcase $j=0$ :

$$
\begin{aligned}
2 p_{0}^{\prime}(r) & =2\left(\sum_{a_{m}^{\prime} \in N\left(p_{0}^{\prime}\right)} a_{m}^{\prime}(r-1)+\sum_{b_{n}^{\prime} \in N\left(p_{0}^{\prime}\right)} b_{n}^{\prime}(r-1)+p_{1}^{\prime}(r-1)\right) \\
& \geqslant 2\left(\sum_{a_{m} \in N\left(p_{0}\right)} a_{m}(r-1)+p_{1}(r-1)\right)=2 p_{0}(r),
\end{aligned}
$$

since $p_{1}^{\prime}(r-1) \geqslant p_{1}(r-1)$, because $1<k / 2$.

Subcase $j=1$ :

$p_{0}^{\prime}(r)+p_{1}^{\prime}(r)=\sum_{a_{m}^{\prime} \in N\left(p_{0}^{\prime}\right)} a_{m}^{\prime}(r-1)+\sum_{b_{n}^{\prime} \in N\left(p_{0}^{\prime}\right)} b_{n}^{\prime}(r-1)+p_{1}^{\prime}(r-1)+p_{0}^{\prime}(r-1)+p_{2}^{\prime}(r-1)$ 


$$
\geqslant \sum_{a_{m} \in N\left(p_{0}\right)} a_{m}(r-1)+p_{1}(r-1)+p_{0}(r-1)+p_{2}(r-1)=p_{0}(r)+p_{1}(r),
$$

since $p_{1}^{\prime}(r-1) \geqslant p_{1}(r-1)$ and $p_{0}^{\prime}(r-1)+p_{2}^{\prime}(r-1) \geqslant p_{0}(r-1)+p_{2}(r-1)$.

Subcase $2 \leqslant \boldsymbol{j} \leqslant \boldsymbol{k}-\mathbf{2}$ : Here we jump back from $r$ to $r-2$, so we need a few notations. Let $d_{A}$ and $d_{B}$ denote the degree of $p_{0}^{\prime}$ in $A$ and $B$, respectively. Furthermore, let $d(v, u)$ denote the distance of the vertices $u$ and $v$. Then

$$
\begin{gathered}
p_{0}^{\prime}(r)+p_{j}^{\prime}(r)=\sum_{a_{m}^{\prime}: d\left(a_{m}^{\prime}, p_{0}^{\prime}\right)=2} a_{m}^{\prime}(r-2)+\sum_{b_{n}^{\prime}: d\left(b_{n}^{\prime}, p_{0}^{\prime}\right)=2} b_{n}^{\prime}(r-2)+\left(d_{A}+d_{B}+1\right) p_{0}^{\prime}(r-2) \\
\quad+p_{2}^{\prime}(r-2)+p_{j-2}^{\prime}(r-2)+2 p_{j}^{\prime}(r-2)+p_{j+2}^{\prime}(r-2) \geqslant \\
\geqslant \sum_{\substack{a_{m}: d\left(a_{m}, p_{0}\right)=2\\
}} a_{m}(r-2)+\left(d_{A}+1\right) p_{0}(r-2)+p_{2}(r-2)+ \\
+p_{j-2}(r-2)+2 p_{j}(r-2)+p_{j+2}(r-2)=p_{0}(r)+p_{j}(r),
\end{gathered}
$$

since the inequality follows from the following inequalities:

$$
\begin{aligned}
a_{m}^{\prime}(r-2) & \geqslant a_{m}(r-2) \\
b_{n}^{\prime}(r-2) & \geqslant 0 \\
\left(d_{B}-1\right) p_{0}^{\prime}(r-2) & \geqslant-p_{0}(r-2) \\
\left(d_{A}-1\right) p_{0}^{\prime}(r-2) & \geqslant\left(d_{A}-1\right) p_{0}(r-2) \\
p_{2}^{\prime}(r-2)+p_{j-2}^{\prime}(r-2) & \geqslant p_{2}(r-2)+p_{j-2}(r-2) \\
2\left(p_{0}^{\prime}(r-2)+p_{j}^{\prime}(r-2)\right) & \geqslant 2\left(p_{0}(r-2)+p_{j}(r-2)\right) \\
p_{0}^{\prime}(r-2)+p_{j+2}^{\prime}(r-2) & \geqslant p_{0}(r-2)+p_{j+2}(r-2) .
\end{aligned}
$$

Subcase $j=k-1$ :

$$
\begin{aligned}
& p_{0}^{\prime}(r)+p_{k-1}^{\prime}(r) \\
= & \sum_{a_{m}^{\prime} \in N\left(p_{0}^{\prime}\right)} a_{m}^{\prime}(r-1)+\sum_{b_{n}^{\prime} \in N\left(p_{0}^{\prime}\right)} b_{n}^{\prime}(r-1)+p_{1}^{\prime}(r-1)+p_{k-2}^{\prime}(r-1)+p_{k}^{\prime}(r-1) \\
= & \sum_{a_{m}: d\left(a_{m}^{\prime}, p_{0}^{\prime}\right)=2} a_{m}^{\prime}(r-2)+d_{A} p_{0}^{\prime}(r-2) \\
& +\sum_{b_{n}^{\prime} \in N\left(p_{0}^{\prime}\right)} b_{n}^{\prime}(r-1)+p_{0}^{\prime}(r-2)+p_{2}^{\prime}(r-2)+p_{k-3}^{\prime}(r-2)+2 p_{k-1}^{\prime}(r-2) .
\end{aligned}
$$

On the other hand,

$$
\begin{aligned}
& p_{0}(r)+p_{k-1}(r) \\
= & \sum_{a_{m} \in N\left(p_{0}\right)} a_{m}(r-1)+p_{1}(r-1)+p_{k-2}(r-1)+p_{k}(r-1)
\end{aligned}
$$




$$
\begin{aligned}
& =\sum_{a_{m}: d\left(a_{m}, p_{0}\right)=2} a_{m}(r-2)+d_{A} p_{0}(r-2)+p_{0}(r-2)+p_{2}(r-2)+p_{k-3}(r-2)+2 p_{k-1}(r-2) \\
& \quad+\sum_{b_{n} \in N\left(p_{k}\right)} b_{n}(r-2) .
\end{aligned}
$$

The inequality $p_{0}^{\prime}(r)+p_{k-1}^{\prime}(r) \geqslant p_{0}(r)+p_{k-1}(r)$ follows from

$$
\begin{aligned}
a_{m}^{\prime}(r-2) & \geqslant a_{m}(r-2) \\
b_{n}^{\prime}(r-1) & \geqslant b_{n}(r-1) \geqslant b_{n}(r-2) \\
\left(d_{A}-1\right) p_{0}^{\prime}(r-2) & \geqslant\left(d_{A}-1\right) p_{0}(r-2) \\
p_{2}^{\prime}(r-2)+p_{k-3}^{\prime}(r-2) & \geqslant p_{2}(r-2)+p_{k-3}(r-2) \\
2\left(p_{0}^{\prime}(r-2)+p_{k-1}^{\prime}(r-2)\right) & \geqslant 2\left(p_{0}(r-2)+p_{k-1}(r-2)\right) .
\end{aligned}
$$

Subcase $j=k$ :

$$
\begin{aligned}
p_{0}^{\prime}(r)+p_{k}^{\prime}(r) & =\sum_{a_{m}^{\prime} \in N\left(p_{0}^{\prime}\right)} a_{m}^{\prime}(r-1)+\sum_{b_{n}^{\prime} \in N\left(p_{0}^{\prime}\right)} b_{n}^{\prime}(r-1)+p_{1}^{\prime}(r-1)+p_{k-1}^{\prime}(r-1) \\
& \geqslant \sum_{a_{m} \in N\left(p_{0}\right)} a_{m}(r-1)+\sum_{b_{n} \in N\left(p_{k}\right)} b_{n}(r-1)+p_{1}(r-1)+p_{k-1}(r-1) \\
& =p_{0}(r)+p_{k}(r) .
\end{aligned}
$$

Proof of the second part of Theorem 1.3. From Lemma 3.2 we only keep the inequalities

$$
\begin{aligned}
a_{m}^{\prime}(r) & \geqslant a_{m}(r) \\
b_{n}^{\prime}(r) & \geqslant b_{n}(r) \\
p_{i}^{\prime}(r) & \geqslant p_{i}(r), p_{k-i}(r) \\
p_{i}^{\prime}(r)+p_{k-i}^{\prime}(r) & \geqslant p_{i}(r)+p_{k-i}(r)
\end{aligned}
$$

for $1 \leqslant m \leqslant M, 1 \leqslant n \leqslant N, 0 \leqslant i \leqslant k / 2$.

For a tree $H$ and $v \in H$, let us write

$$
\begin{aligned}
\mathbf{h}(H, v, T) & =\left(a_{1}, a_{2}, \ldots, a_{M}, p_{0}, p_{1}, \ldots, p_{k}, b_{1}, b_{2}, \ldots, b_{N}\right) \text { and } \\
\mathbf{h}\left(H, v, T^{\prime}\right) & =\left(a_{1}^{\prime}, a_{2}^{\prime}, \ldots, a_{M}^{\prime}, p_{0}^{\prime}, p_{1}^{\prime}, \ldots, p_{k}^{\prime}, b_{1}^{\prime}, b_{2}^{\prime}, \ldots, b_{N}^{\prime}\right),
\end{aligned}
$$

where we use the labels of vertices of $T$ and $T^{\prime}$ to index the parameters of the hom-vectors to $T$ and $T^{\prime}$, respectively. We say that $\mathbf{h}(H, v, T) \leqslant \mathbf{h}\left(H, v, T^{\prime}\right)$ if the following inequalities hold

$$
\begin{gathered}
a_{m}^{\prime} \geqslant a_{m} \\
b_{n}^{\prime} \geqslant b_{n}
\end{gathered}
$$

The ELECtronic Journal of COMbinatorics 21(4) (2014), \#P4.9 


$$
\begin{aligned}
p_{i}^{\prime}+p_{k-i}^{\prime} & \geqslant p_{i}+p_{k-i} \\
p_{i}^{\prime} & \geqslant p_{i} \\
p_{i}^{\prime} & \geqslant p_{k-i}
\end{aligned}
$$

for $1 \leqslant m \leqslant M, 1 \leqslant n \leqslant N, 0 \leqslant i \leqslant k / 2$. As we have seen these inequalities hold for a path $P_{r}$ and its endvertex. Since these inequalities are preserved for Hadamard-product by Lemma 3.1, we see that $\mathbf{h}(H, v, T) \leqslant \mathbf{h}\left(H, v, T^{\prime}\right)$ for starlike trees $H$, where $v$ is the center of the starlike tree. This implies that

$$
\operatorname{hom}\left(H, T^{\prime}\right) \geqslant \operatorname{hom}(H, T)
$$

The following generalization of inequality (1.2) follows immediately from Proposition 1.1 and Theorem 1.3.

Corollary 3.3. Let $H$ be a starlike tree and let $T_{n}$ be a tree on $n$ vertices. Then

$$
\operatorname{hom}\left(H, P_{n}\right) \leqslant \operatorname{hom}\left(H, T_{n}\right) \leqslant \operatorname{hom}\left(H, S_{n}\right) .
$$

The reader may wonder that if inequality (1.4) holds when $k$ is odd and $H$ is not a starlike tree. This is not true in general. A counterexample will be constructed in the following, which also shows that

$$
\operatorname{hom}\left(H, T_{n}\right) \leqslant \operatorname{hom}\left(H, S_{n}\right)
$$

is not true for any tree $H$.

Proposition 3.4. Let $T$ be a tree with color classes $A$ and $B$ considered as a bipartite graph. Then

$$
\operatorname{hom}\left(T, S_{n}\right)=(n-1)^{|A|}+(n-1)^{|B|} .
$$

Corollary 3.5. Let $T_{m}$ be a tree on $m$ vertices, then

$$
\operatorname{hom}\left(P_{m}, S_{n}\right) \leqslant \operatorname{hom}\left(T_{m}, S_{n}\right) \leqslant \operatorname{hom}\left(S_{m}, S_{n}\right)
$$

If $T \neq S_{m}$ then the second inequality is strict.

Proof of Proposition 3.4. Since $T$ and $S_{n}$ are bipartite graphs, a color class of $T$ have to go into a color class of $S_{n}$. If the color class $A$ goes to the center of $S_{n}$, then any vertex belonging to the color class of $B$ can go to any leaf of the star, so it provides $(n-1)^{|B|}$ homomorphisms. The other case provides $(n-1)^{|A|}$ homomorphisms.

This simple proposition also shows us how to construct a tree $T_{n}$ for which $\operatorname{hom}\left(T_{n}, T_{n}\right)>\operatorname{hom}\left(T_{n}, S_{n}\right)$.

Let $T_{n}=S_{2 k}^{*}$ be the doublestar on $2 k$ vertices with $2 k-2$ leaves and two vertices of degree $k$. Then it is easy to see that

$$
\operatorname{hom}\left(S_{2 k}^{*}, S_{2 k}^{*}\right)>2(k-1)^{2(k-1)}=2\left(k^{2}-2 k+1\right)^{k-1},
$$




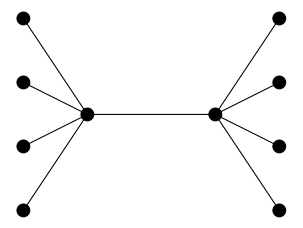

Figure 6: The doublestar $S_{10}^{*}$.

while

$$
\operatorname{hom}\left(S_{2 k}^{*}, S_{2 k}\right)=2(2 k-1)^{k}
$$

Hence for $k \geqslant 5$ we have

$$
\operatorname{hom}\left(S_{2 k}^{*}, S_{2 k}^{*}\right)>\operatorname{hom}\left(S_{2 k}^{*}, S_{2 k}\right) .
$$

Note that $S_{2 k}$ can be obtained from $S_{2 k}^{*}$ by a KC-transformation.

\section{Graph homomorphisms from trees}

\subsection{Markov chains and homomorphisms}

Theorem 4.1. Let $G$ be a graph and let $P=\left(p_{i j}\right)$ be a Markov chain on $G$ :

$$
\sum_{j \in N(i)} p_{i j}=1 \quad \text { for all } i \in V(G),
$$

where $p_{i j} \geqslant 0$ and $p_{i j}=0$ if $(i, j) \notin E(G)$. Let $Q=\left(q_{i}\right)$ be the stationary distribution of $P$ :

$$
\sum_{j \in N(i)} q_{j} p_{j i}=q_{i} \quad \text { for all } i \in V(G) .
$$

Let us define the following entropies:

$$
H(Q)=\sum_{i \in V(G)} q_{i} \log \frac{1}{q_{i}},
$$

and

$$
H(D \mid Q)=\sum_{i \in V(G)} q_{i} \log d_{i}
$$

where $d_{i}$ is the degree of the vertex $i$, and let

$$
H(P \mid Q)=\sum_{i \in V(G)} q_{i}\left(\sum_{j \in N(i)} p_{i j} \log \frac{1}{p_{i j}}\right) .
$$

Let $T_{m}$ be a tree with $\ell$ leaves on $m$ vertices, where $m \geqslant 3$. Then

$$
\operatorname{hom}\left(T_{m}, G\right) \geqslant \exp (H(Q)+\ell H(D \mid Q)+(m-1-\ell) H(P \mid Q)) .
$$


Proof. Let $v$ be a root of $T$. Let $a_{i}$ be the number of homomorphisms of $T_{m}$ into $G$ such that the root vertex $v$ goes into the vertex $i \in V(G)$. Let

$$
F\left(T_{m}(v), G\right)=\prod_{i=1}^{n} a_{i}^{q_{i}} .
$$

We will show by induction on $m$ that

$$
F\left(T_{m}(v), G\right) \geqslant \exp \left(\ell^{*} H(D \mid Q)+\left(m-1-\ell^{*}\right) H(P \mid Q)\right),
$$

where $\ell^{*}$ is the number of leaves different from $v$, so it is $\ell$ if $v$ is not a leaf and $\ell-1$ if $v$ is a leaf. Note that

$$
F\left(K_{2}(v), G\right)=\exp H(D \mid Q) .
$$

If $v$ is not a leaf of $T_{m}$, then we can decompose $T_{m}$ to $T_{1}(v)$ and $T_{2}(v)$. Then

$$
F\left(T_{m}(v), G\right)=F\left(T_{1}(v), G\right) F\left(T_{2}(v), G\right)
$$

because of the Hadamard-products of the hom-vectors. From this the claim follows immediately by induction.

If $v$ is a leaf of $T_{m}$ with the unique neighbor $u$, then let

$$
\mathbf{h}\left(T_{m}-v, u, G\right)=\left(b_{1}, \ldots, b_{n}\right) .
$$

So $a_{i}=\sum_{j \in N(i)} b_{j}$.

For positive numbers $r_{1}, \ldots, r_{t}$ and positive weights $w_{1}, \ldots, w_{t}$ with $\sum_{i=1}^{t} w_{i}=1$, the weighted AM-GM inequality says that

$$
\begin{aligned}
r_{1}+\cdots+r_{t} & =w_{1}\left(\frac{r_{1}}{w_{1}}\right)+\cdots+w_{t}\left(\frac{r_{t}}{w_{t}}\right) \\
& \geqslant\left(\frac{r_{1}}{w_{1}}\right)^{w_{1}} \cdots\left(\frac{r_{t}}{w_{t}}\right)^{w_{t}} \\
& =\exp \left(\sum_{i=1}^{t} w_{i} \log \frac{1}{w_{i}}\right) \prod_{i=1}^{t} r_{i}^{w_{i}} .
\end{aligned}
$$

Hence

$$
\begin{aligned}
F\left(T_{m}(v), G\right) & =\prod_{i=1}^{n} a_{i}^{q_{i}}=\prod_{i=1}^{n}\left(\sum_{j \in N(i)} b_{j}\right)^{q_{i}} \\
& \geqslant \prod_{i=1}^{n}\left(\prod_{j \in N(i)}\left(\frac{b_{j}}{p_{i j}}\right)^{p_{i j}}\right)^{q_{i}} \\
& =\prod_{i=1}^{n}\left(\prod_{j \in N(i)}\left(\frac{1}{p_{i j}}\right)^{p_{i j} q_{i}}\right) \prod_{i=1}^{n} b_{i}^{\sum_{j \in N(i)} p_{j i} q_{j}}
\end{aligned}
$$

The electronic journal of Combinatorics 21(4) (2014), \#P4.9 


$$
=\prod_{i=1}^{n}\left(\prod_{j \in N(i)}\left(\frac{1}{p_{i j}}\right)^{p_{i j} q_{i}}\right) \prod_{i=1}^{n} b_{i}^{q_{i}} .
$$

In the last step we used that $Q$ is a stationary distribution with respect to $P$. Hence

$$
F\left(T_{m}(v), G\right) \geqslant \exp (H(P \mid Q)) F\left(\left(T_{m}-v\right)(u), G\right) .
$$

Now the claim follows by induction.

To finish the proof of the theorem, we only have to choose a nonleaf root and use that

$$
\operatorname{hom}\left(T_{m}, G\right)=\sum_{i=1}^{n} a_{i} \geqslant \exp (H(Q)) F\left(T_{m}(v), G\right) .
$$

Remark 4.2. Note that the inequality $H(D \mid Q) \geqslant H(P \mid Q)$ always holds. Consequently,

$$
\operatorname{hom}\left(T_{m}, G\right) \geqslant \exp (H(Q)+(m-1) H(P \mid Q)) \text {. }
$$

As Theorem 4.1 suggests, this is an inequality for entropies and indeed, it can be proved in this way. By $P$ and $Q$, we defined a distribution on the set of homomorphisms: we choose a root according to $Q$, then we choose every nonleaf new vertex according to $P$ and finally we choose the leaves uniformly. The entropy of this distribution is exactly $H(Q)+\ell H(D \mid Q)+(m-1-\ell) H(P \mid Q)$ since every nonleaf vertex has distribution $Q$. Note that this entropy is smaller than the entropy of the uniform distribution, that is, $\log \operatorname{hom}\left(T_{m}, G\right)$. For basic facts about entropy, see for example [5].

Theorem 4.3. Let $G$ be a connected graph on the vertex set $\{1,2, \ldots, n\}$ and let $\lambda$ be the largest eigenvalue of the adjacency matrix of the graph $G$. Let y be a positive eigenvector of unit length corresponding to $\lambda$. Let $q_{i}=y_{i}^{2}$. Then for any rooted tree $T_{m}$ on $m$ vertices we have

$$
\operatorname{hom}\left(T_{m}, G\right) \geqslant \exp \left(H_{\lambda}(G)\right) \lambda^{m-1},
$$

where

$$
H_{\lambda}(G)=\sum_{i=1}^{n} q_{i} \log \frac{1}{q_{i}}
$$

is the spectral entropy of the graph $G$.

Proof. We will use Theorem 4.1. Let $p_{i j}=\frac{y_{j}}{\lambda y_{i}}$. Since $\underline{y}$ is a positive eigenvector, we have $p_{i j}>0$. For all $i$ we have $\lambda y_{i}=\sum_{j \in N(i)} y_{j}$, thus $\sum_{j \in N(i)} p_{i j}=1$. For $q_{i}=y_{i}^{2}$ we have

$$
q_{i} p_{i j}=y_{i}^{2} \frac{y_{j}}{\lambda y_{i}}=\frac{1}{\lambda} y_{i} y_{j}=q_{j} p_{j i}
$$

Hence

$$
\sum_{i \in N(j)} q_{j} p_{j i}=\sum_{i \in N(j)} q_{i} p_{i j}=q_{i}
$$


This means that $P=\left(p_{i j}\right)$ is a Markov chain with stationary distribution $Q=\left(q_{i}\right)$. The conditional entropy

$$
\begin{aligned}
H(P \mid Q) & =\sum_{i \in V(G)} q_{i}\left(\sum_{j \in N(i)} p_{i j} \log \frac{1}{p_{i j}}\right) \\
& =\sum_{i \in V(G)} y_{i}^{2}\left(\sum_{j \in N(i)} \frac{y_{j}}{\lambda y_{i}} \log \frac{\lambda y_{i}}{y_{j}}\right) \\
& =\sum_{\{i, j\} \in E(G)} \frac{y_{i} y_{j}}{\lambda}\left(2 \log \lambda+\log \frac{y_{i}}{y_{j}}+\log \frac{y_{j}}{y_{i}}\right) \\
& =\log (\lambda) \frac{1}{\lambda} \sum_{(i, j) \in E(G)} y_{i} y_{j}=\log \lambda .
\end{aligned}
$$

Hence the result follows from Theorem 4.1.

Remark 4.4. A Markov chain is called reversible if $q_{i} p_{i j}=q_{j} p_{j i}$ for all $i, j \in V(G)$. As we have seen, the Markov chain constructed in the previous proof is reversible. It is not hard to show that on trees every Markov chain is reversible.

Remark 4.5. Theorem 4.3 is the best possible in the sense that there cannot be a larger number than $\lambda$ in such a statement since

$$
\operatorname{hom}\left(P_{m}, G\right) \leqslant n \lambda^{m-1} \text {. }
$$

Indeed,

$$
\frac{\operatorname{hom}\left(P_{m}, G\right)}{n}=\frac{\mathbf{1}_{\mathbf{n}}{ }^{T} A^{m-1} \mathbf{1}_{\mathbf{n}}}{\mathbf{1}_{\mathbf{n}}{ }^{T} \mathbf{1}_{\mathbf{n}}} \leqslant \max _{v \neq \underline{0}} \frac{v^{T} A^{m-1} v}{v^{T} v}=\lambda_{\max }\left(A^{m-1}\right)=\lambda^{m-1} .
$$

Note that we can deduce that if $\left(T_{m}\right)_{m=1}^{\infty}$ is a sequence of trees such that $T_{m}$ has $m$ vertices then

$$
\liminf _{m \rightarrow \infty} \operatorname{hom}\left(T_{m}, G\right)^{1 / m} \geqslant \liminf _{m \rightarrow \infty} \operatorname{hom}\left(P_{m}, G\right)^{1 / m}=\lambda .
$$

This result could have been deduced as well from a theorem of B. Rossman and E. Vee [21] claiming that

$$
\operatorname{hom}\left(T_{m}, G\right) \geqslant \operatorname{hom}\left(C_{m}, G\right),
$$

where $C_{m}$ is the cycle on $m$ vertices. In fact, this was proved for directed trees and cycles, but it implies the inequality for undirected tree and cycle. This result can also be deduced from Theorem 3.1 of [13].

The following special case of Theorem 4.1, involving the degree sequence of graphs, is Theorem 3 in the paper [9]. 
Theorem 4.6 (Dellamonica et al.). Let $G$ be a graph on the vertex set $\{1,2, \ldots, n\}$ with $e(G)$ edges and with degree sequence $\left(d_{1}, \ldots, d_{n}\right)$. Then for any tree $T_{m}$ on $m$ vertices we have

$$
\operatorname{hom}\left(T_{m}, G\right) \geqslant 2 e(G) \cdot C^{m-2}
$$

where

$$
C=\left(\prod_{i=1}^{n} d_{i}^{d_{i}}\right)^{1 / 2 e(G)}
$$

Proof. Let us consider the following classical Markov chain: $p_{i j}=\frac{1}{d_{i}}$ if $j \in N(i)$. The stationary distribution is $q_{i}=\frac{d_{i}}{2 e(G)}$. Note that

$$
H(P \mid Q)=\sum_{i \in V(G)} q_{i}\left(\sum_{j \in N(i)} p_{i j} \log \frac{1}{p_{i j}}\right)=\sum_{i \in V(G)} q_{i} \log d_{i}=\frac{1}{2 e(G)} \sum_{i \in V(G)} d_{i} \log d_{i}=\log C
$$

and

$$
H(Q)+H(P \mid Q)=\sum_{(i, j) \in E(G)} q_{i} p_{i j} \log \frac{1}{q_{i} p_{i j}}=\sum_{(i, j) \in E(G)} \frac{1}{2 e(G)} \log (2 e(G))=\log (2 e(G)) .
$$

Hence the result follows from Theorem 4.1.

Definition 4.7. The homomorphism density $t(H, G)$ is defined as follows:

$$
t(H, G)=\frac{\operatorname{hom}(H, G)}{|V(G)|^{|V(H)|}} .
$$

This is the probability that a random map is a homomorphism.

Sidorenko's conjecture says that

$$
t(H, G) \geqslant t\left(K_{2}, G\right)^{e(H)}
$$

for every bipartite graph $H$ with $e(H)$ edges. It is known that Sidorenko's conjecture [18] is true for trees. By now, there are many proofs for this particular case of Sidorenko's conjecture: see $[11,15]$ and it can be deduced as well from Theorem 3.1 of [13]. Below we give a new proof for this fact.

Theorem 4.8. For any tree $T_{m}$ on $m$ vertices and a graph $G$ we have

$$
t\left(T_{m}, G\right) \geqslant t\left(K_{2}, G\right)^{m-1} .
$$

Proof. Let $|V(G)|=n$. The theorem will immediately follows form Theorem 4.6. By convexity of the function $x \log x$ we have

$$
\frac{1}{2 e(G)} \sum_{i \in V(G)} d_{i} \log d_{i} \geqslant \frac{1}{2 e(G)} n\left(\frac{2 e(G)}{n} \log \frac{2 e(G)}{n}\right)=\log \frac{2 e(G)}{n} .
$$

Hence

$$
t\left(T_{m}, G\right)=\frac{\operatorname{hom}\left(T_{m}, G\right)}{n^{m}} \geqslant \frac{1}{n^{m}} 2 e(G)\left(\frac{2 e(G)}{n}\right)^{m-2}=\left(\frac{2 e(G)}{n^{2}}\right)^{m-1}=t\left(K_{2}, G\right)^{m-1} .
$$




\subsection{Sidorenko's theorem on extremality of stars}

The objective of this section is to give a new proof for Theorem 1.5 in order to keep this paper self-contained. This was proved originally by Sidorenko [19]. Our proof is very similar to the original one, but it is slightly more elementary.

Before we start the proof we will need two definitions and two lemmas.

Definition 4.9. Let $M_{u}$ and $N_{v}$ be two rooted graphs with root vertices $u$ and $v$, respectively. Then $M_{u} \circ_{u=v} N_{v}$ denotes the graph obtained from $M_{u} \cup N_{v}$ by identifying the vertices $u$ and $v$.

Lemma 4.10. Let $R_{u, v}$ be a graph with specified (not necessarily distinct) vertices $u$ and $v$. Let $J_{u^{\prime}}$ and $K_{v^{\prime}}$ be two graphs with root vertices $u^{\prime}$ and $v^{\prime}$. Finally, let the graphs $A, B$ and $C$ be obtained from $R_{u, v}, J_{u^{\prime}}, K_{v^{\prime}}$ as follows:

$$
\begin{aligned}
& A=\left(R_{u, v} \circ_{u=u^{\prime}} J_{u^{\prime}}\right) \circ_{v=v^{\prime}} K_{v^{\prime}} \text {, } \\
& B=\left(R_{u, v} \circ_{u=u^{\prime}} J_{u^{\prime}}\right) \circ_{u=u^{\prime}} J_{u^{\prime}} \text {, } \\
& C=\left(R_{u, v} \circ_{v=v^{\prime}} K_{v^{\prime}}\right) \circ_{v=v^{\prime}} K_{v^{\prime}} \text {. }
\end{aligned}
$$

(In other words, in $B$ and $C$ we attach two copies of the same graph at the specified vertex.) Then for any graph $G$ we have

$$
2 \operatorname{hom}(A, G) \leqslant \operatorname{hom}(B, G)+\operatorname{hom}(C, G) .
$$

Proof. Let $i, j \in V(G)$ and let $h\left(R_{u, v}, i, j\right)$ denote the number of homomorphisms of $R_{u, v}$ to $G$ where $u$ goes to $i$ and $v$ goes to $j$. We similarly define $h\left(J_{u^{\prime}}, i\right)$ and $h\left(K_{v^{\prime}}, j\right)$. Then

$$
\operatorname{hom}(A, G)=\sum_{i, j \in V(G)} h\left(R_{u, v}, i, j\right) h\left(J_{u^{\prime}}, i\right) h\left(K_{v^{\prime}}, j\right)
$$

Similarly,

$$
\operatorname{hom}(B, G)=\sum_{i, j \in V(G)} h\left(R_{u, v}, i, j\right) h\left(J_{u^{\prime}}, i\right)^{2}
$$

and

$$
\operatorname{hom}(C, G)=\sum_{i, j \in V(G)} h\left(R_{u, v}, i, j\right) h\left(K_{v^{\prime}}, j\right)^{2} .
$$

Hence

$$
\begin{aligned}
& \operatorname{hom}(B, G)+\operatorname{hom}(C, G)-2 \operatorname{hom}(A, G) \\
= & \sum_{i, j \in V(G)} h\left(R_{u, v}, i, j\right)\left(h\left(J_{u^{\prime}}, i\right)-h\left(K_{v^{\prime}}, j\right)\right)^{2} \geqslant 0 .
\end{aligned}
$$

We are done. 
Definition 4.11. Let $d(u, v)$ be the distance of the vertices $u, v \in V(G)$. Then the Wiener-index $W(G)$ of a graph $G$ is defined as

$$
W(G):=\sum_{u, v \in V(G)} d(u, v)
$$

In our application $R_{u, v}$ will be a tree and $J_{u^{\prime}}$ and $K_{v^{\prime}}$ be the trees on 2 vertices. The following lemma about the Wiener-index is trivial.

Lemma 4.12. Let $R_{u, v}$ be a tree with distinct vertices $u$ and $v$. Let $J_{u^{\prime}}$ and $K_{v^{\prime}}$ be two copies of the two-node trees with root vertices $u^{\prime}$ and $v^{\prime}$, respectively. Finally, let the graphs $A, B$ and $C$ be obtained from $R_{u, v}, J_{u^{\prime}}, K_{v^{\prime}}$ as in the former lemma. Then $2 W(A)>W(B)+W(C)$.

Proof of Theorem 1.5. Let $\mathcal{T}_{G}$ be the set of those trees $F$ on $m$ vertices for which $\operatorname{hom}(F, G)$ is maximal. Let $T \in \mathcal{T}_{G}$ be the tree for which $W(T)$ is minimal. We show that $T=S_{m}$. Assume for contradiction that $T \neq S_{m}$. Then $T$ has two leaves, $a$ and $b$ such that $d(a, b) \geqslant 3$. Let $u$ and $v$ be the unique neighbors of $a$ and $b$, respectively. Then $u \neq v$. Let $R_{u, v}=T-\{a, b\}, J_{u^{\prime}}=\left\{u^{\prime}, a\right\}$ and $K_{v^{\prime}}=\left\{v^{\prime}, b\right\}$. Then

$$
A=\left(R_{u, v} \circ_{u=u^{\prime}} J_{u^{\prime}}\right) \circ_{v=v^{\prime}} K_{v^{\prime}}=T .
$$

As in the lemmas, let

$$
\begin{aligned}
& B=\left(R_{u, v} \circ_{u=u^{\prime}} J_{u^{\prime}}\right) \circ_{u=u^{\prime}} J_{u^{\prime}}, \\
& C=\left(R_{u, v} \circ_{v=v^{\prime}} K_{v^{\prime}}\right) \circ_{v=v^{\prime}} K_{v^{\prime}} .
\end{aligned}
$$

Note that $B$ and $C$ are also trees on $m$ vertices. By the Lemma we have

$$
2 \operatorname{hom}(A, G) \leqslant \operatorname{hom}(B, G)+\operatorname{hom}(C, G) .
$$

Since $A=T \in \mathcal{T}_{G}$, then $\operatorname{hom}(B, G)+\operatorname{hom}(C, G) \leqslant 2 \operatorname{hom}(A, G)$. So $\operatorname{hom}(A, G)=$ $\operatorname{hom}(B, G)=\operatorname{hom}(C, G)$ implying that $B, C \in \mathcal{T}_{G}$ as well. But then $2 W(T)>W(B)+$ $W(C)$, so one of them has strictly smaller Wiener-index than $T$, this contradicts the choice of $T$. Hence $T$ must be $S_{m}$.

Remark 4.13. Let $E_{7}$ be the tree obtained from $P_{6}$ by putting a pendant edge to the third vertex of the path. Then there is a tree $T$ for which

$$
\operatorname{hom}\left(P_{7}, T\right)>\operatorname{hom}\left(E_{7}, T\right) .
$$

The following tree $T$ is suitable: let $T=T\left(k_{1}, k_{2}, k_{3}\right)$ be the tree where the root vertex $v_{0}$ have $k_{1}$ neighbors, all of its neighbors has $k_{2}+1$ neighbors and the vertices having distance 2 from $v_{0}$ have $k_{3}+1$ neighbors. If we choose $k_{1}, k_{2}, k_{3}$ such that $k_{2} \ll k_{1} \ll k_{3} \ll k_{1} k_{2}$ (for instance $k_{i}=k^{\alpha_{i}}$, where $\alpha_{2}<\alpha_{1}<\alpha_{3}<\alpha_{1}+\alpha_{2}$ and $k$ is large), then

$$
\operatorname{hom}\left(P_{7}, T\right)-\operatorname{hom}\left(E_{7}, T\right)=k_{1}^{2} k_{2}^{2} k_{3}^{2}+o\left(k_{1}^{2} k_{2}^{2} k_{3}^{2}\right) .
$$




\section{Proofs of Theorems 1.6 and 1.8}

In this section we give the proof of Theorem 1.6 and Theorem 1.8. As we will see, Theorem 1.6 with some additional observations implies Theorem 1.8.

To prove Theorem 1.6 we will build on the fact that there are not many homomorphisms into a path. Indeed, by Theorem 1.5 we have

$$
\operatorname{hom}\left(T_{m}, P_{n}\right) \leqslant \operatorname{hom}\left(S_{m}, P_{n}\right)=(n-2) 2^{m-1}+2 .
$$

So for a particular tree $T_{n}$, it is enough to prove that for every tree $T_{m}$ we have

$$
\operatorname{hom}\left(T_{m}, T_{n}\right) \geqslant(n-2) 2^{m-1}+2 .
$$

This would immediately imply that

$$
\operatorname{hom}\left(T_{m}, T_{n}\right) \geqslant \operatorname{hom}\left(T_{m}, P_{n}\right) .
$$

We will prove that inequality 5.1 is indeed true for all trees $T_{n}$ with at least four leaves and for a large class of trees with three leaves. For the remaining trees with three leaves we use Theorem 1.3.
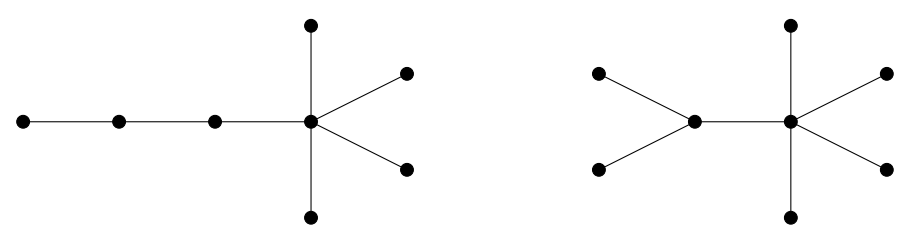

Figure 7: The trees $T_{8}$ (left) and $T_{8}^{\prime}$ (right).

Remark 5.1. To prove Theorem 1.6 and Theorem 1.8 we cannot rely entirely on the use of KC-transformation. That is why we had to find another strategy to prove these theorems.

Indeed, KC-transformation does not always increase the number of endomorphisms of trees. The first counterexample is the two trees on 8 vertices in Fig. 7 . The tree $T_{8}^{\prime}$ is the KC-transformation of $T_{8}$, but $\left|\operatorname{End}\left(T_{8}^{\prime}\right)\right|=10430<17190=\left|\operatorname{End}\left(T_{8}\right)\right|$.

\subsection{The extremality of the star}

Note that Theorem 1.5 and Theorem 1.3 together implies the following chain of inequalities:

$$
\left|\operatorname{End}\left(T_{n}\right)\right|=\operatorname{hom}\left(T_{n}, T_{n}\right) \leqslant \operatorname{hom}\left(S_{n}, T_{n}\right) \leqslant \operatorname{hom}\left(S_{n}, S_{n}\right)=\left|\operatorname{End}\left(S_{n}\right)\right|,
$$

since $S_{n}$ is a starlike tree. In this section, we will also give a direct proof for it.

Theorem 1.8 (Second part). Let $T_{n}$ be a tree on $n$ vertices. Then

$$
\left|\operatorname{End}\left(T_{n}\right)\right| \leqslant\left|\operatorname{End}\left(S_{n}\right)\right| \text {. }
$$

If $T_{n} \neq S_{n}$ then strict inequality holds. 
Proof. For the sake of simplicity we prove the statement for $n \geqslant 17$. The same proof applies to $n<17$, we only need to compute a bit more carefully. In the end of the proof we will give the details of this more precise calculation.

Note that $\left|\operatorname{End}\left(S_{n}\right)\right|=(n-1)^{n-1}+(n-1)$.

Let $T_{n}$ be a tree on $n$ vertices and let $d=d_{1} \geqslant d_{2} \geqslant \ldots \geqslant d_{n}$ be its degree sequence. Note that $d_{1}+d_{2} \leqslant n$, since the tree has only $n-1$ edges and the stars corresponding to the first two largest degrees can share at most one common edge.

First we prove that $\left|\operatorname{End}\left(T_{n}\right)\right| \leqslant n d^{n-1}$. To see it, let $u_{1}, \ldots u_{n}$ be the vertices of the tree $T_{n}$ such that $u_{1}, \ldots, u_{k}$ induces a tree for every $k$. Then we can chose the image of $u_{1}$ by $n$ ways, and if we have already chosen the image of $u_{1}, \ldots, u_{k-1}$, then we can chose the image of $u_{k}$ in at most $d$ ways, since it must be the neighbor of some previous vertex. This means that $\left|\operatorname{End}\left(T_{n}\right)\right| \leqslant n d^{n-1}$.

If $d \leqslant 2 n / 3$ then

$$
n d^{n-1} \leqslant n\left(\frac{2 n}{3}\right)^{n} \leqslant(n-1)^{n-1}
$$

if $n \geqslant 17$, since then

$$
\left(\frac{3}{2}\right)^{n} \geqslant e n^{2} \geqslant n^{2}\left(1+\frac{1}{n-1}\right)^{n-1}
$$

So we can assume that $d \geqslant \frac{2 n}{3}$. Set $d=n-k$. We can assume that $T_{n} \neq S_{n}$, consequently $k \geqslant 2$. Let $v_{1}$ be the vertex having the largest degree and $v_{2}, \ldots, v_{d+1}$ its neighbors. Now we can decompose the set of endomorphisms according to the image of $v_{1}$ is $v_{1}$ or not. If it is $v_{1}$ then there can be at most $d^{n-1}$ such endomorphisms. If the image of $v_{1}$ is not $v_{1}$, then we can chose that image in at most $(n-1)$ ways and the image of $v_{2}, v_{3}, \ldots, v_{d+1}$ can be chosen at most $d_{2}$ times and the image of all other vertices can be chosen in at most $d$ ways. Hence

$$
\left|\operatorname{End}\left(T_{n}\right)\right| \leqslant d^{n-1}+(n-1) d_{2}^{d} d^{n-1-d} .
$$

All we need to prove is that if $d \leqslant n-2$ then

$$
d^{n-1}+(n-1) d_{2}^{d} d^{n-1-d} \leqslant(n-1)^{n-1}+(n-1) .
$$

With the notations $d=n-k$ we have

$$
d^{n-1}+(n-1) d_{2}^{d} d^{n-1-d} \leqslant(n-k)^{n-1}+(n-1) k^{n-k}(n-k)^{k-1} .
$$

By the binomial theorem we have

$$
(n-1)^{n-1}=(n-k+k-1)^{n-1} \geqslant(n-k)^{n-1}+(n-1)(n-k)^{n-2}(k-1) .
$$

It is enough to prove that $(n-k)^{n-2} \geqslant k^{n-k}(n-k)^{k-1}$. This is equivalent with

$$
(n-k)^{n-k-1} \geqslant k^{n-k}
$$


and it is true since it is equivalent with

$$
\left(\frac{n}{k}-1\right)^{n-k} \geqslant 2^{n-k} \geqslant n-k
$$

In the last step we have used that $n / k \geqslant 3$.

It is clear from the proof that we only have to check whether one of the inequalities hold for some $d$ :

$$
n \leqslant\left(\frac{n-1}{d}\right)^{n-1} \text { or }\left(\frac{n}{k}-1\right)^{n-k} \geqslant n-k .
$$

For $8 \leqslant n \leqslant 16$ it is easy to see that if $d \leqslant n-4$ then the first inequality holds and if $d>n-4$, equivalently $k \leqslant 3$ then the second inequality holds. For $n=5,6,7$ the first inequality holds if $d \leqslant n-3$, and the second inequality holds if $d>n-3$, equivalently $k \leqslant 2$. For $n=4$ the claim is trivial $30=\left|\operatorname{End}\left(S_{4}\right)\right|>\left|\operatorname{End}\left(P_{4}\right)\right|=16$.

\subsection{The extremality of the path}

Theorem 5.2. Let $T_{m}$ and $T_{n}$ be trees on $m$ and $n$ vertices, respectively. If the tree $T_{n}$ has at least four leaves, then

$$
\operatorname{hom}\left(T_{m}, T_{n}\right) \geqslant(n-2) 2^{m-1}+2 .
$$

An easy consequence of this theorem is the following.

Corollary 5.3. If $T_{n}$ is a tree on $n$ vertices with at least 4 leaves, then

$$
\operatorname{hom}\left(T_{m}, T_{n}\right) \geqslant \operatorname{hom}\left(T_{m}, P_{n}\right) .
$$

Proof. Indeed,

$$
\operatorname{hom}\left(T_{m}, T_{n}\right) \geqslant(n-2) 2^{m-1}+2=\operatorname{hom}\left(S_{m}, P_{n}\right) \geqslant \operatorname{hom}\left(T_{m}, P_{n}\right),
$$

where the second inequality follows from Theorem 1.5.

A consequence of this theorem and Theorem 1.4 (the proof of which will be given in the follow-up paper [8]) is that path has the minimal number of endomorphisms.

Theorem 1.8(First part). For all trees $T_{n}$ on $n$ vertices we have

$$
\left|\operatorname{End}\left(T_{n}\right)\right| \geqslant\left|\operatorname{End}\left(P_{n}\right)\right| \text {. }
$$

Proof. If $T_{n}$ has at least four leaves, then

$$
\operatorname{hom}\left(T_{n}, T_{n}\right) \geqslant \operatorname{hom}\left(T_{n}, P_{n}\right) \geqslant \operatorname{hom}\left(P_{n}, P_{n}\right),
$$

where the first inequality follows from Corollary 5.3, while the second inequality follows from Theorem 1.4. If the tree $T_{n}$ has exactly three leaves, then it is star-like. Hence we can use Theorem 1.3 to prove the first inequality:

$$
\operatorname{hom}\left(T_{n}, T_{n}\right) \geqslant \operatorname{hom}\left(T_{n}, P_{n}\right) \geqslant \operatorname{hom}\left(P_{n}, P_{n}\right) .
$$


The proof of Theorem 5.2 will be given next, which would complete the proof of Theorem 1.8.

First, we prove a reduction lemma which says that we only have to prove Theorem 5.2 for trees with exactly 4 leaves.

Lemma 5.4 (Reduction lemma). Let $T_{m}$ be a tree on $m$ vertices and let $n$ be fixed. Assume that for any tree $T_{k}$ we have

$$
\operatorname{hom}\left(T_{m}, T_{k}\right) \geqslant(k-2) 2^{m-1}+2,
$$

where $k<n$ and $T_{k}$ has at least four leaves, or $k=n$ and $T_{k}$ has exactly four leaves. Then for any tree $T_{n}$ on $n$ vertices with at least 4 leaves we have

$$
\operatorname{hom}\left(T_{m}, T_{n}\right) \geqslant(n-2) 2^{m-1}+2 .
$$

In the proof of this lemma we will subsequently use the following very simple fact.

Fact. If $G$ is a graph and $G_{1}, G_{2}$ are induced subgraphs of $G$ with possible intersection, then for any graph $H$ we have

$$
\operatorname{hom}(H, G) \geqslant \operatorname{hom}\left(H, G_{1}\right)+\operatorname{hom}\left(H, G_{2}\right)-\operatorname{hom}\left(H, G_{1} \cap G_{2}\right) .
$$

Proof of the lemma. We can assume that $m \geqslant 2$. Assume that $T_{n}$ is a tree with at least 5 leaves. Otherwise we have nothing to prove.

Let us call a path maximal in $T_{n}$ if it connects leaves. If a maximal path contains $k$ vertices of degree at least 3 , then we say that the maximal path has $k$ branches.

Case 1: $\boldsymbol{T}_{n}$ contains a maximal path with at least 3 branches. Let $v_{0} P v_{r}$ be a maximal path with vertices $u_{1}, \ldots, u_{k}$ having degree at least 3 . Let $B_{1}, \ldots, B_{k}$ be the branches which we get if we delete all vertices and edges of the path $v_{0} P v_{r}$ except $u_{1}, \ldots, u_{k}$. So $B_{i}$ is a rooted tree with root $u_{i}$. Let $u_{2}^{-}$and $u_{2}^{+}$be the two neighbors of $u_{2}$ on the path $v_{0} P v_{r}$. Let $T^{(2)}$ be the tree induced by the vertices $V\left(B_{2}\right) \cup\left\{u_{2}^{-}, u_{2}^{+}\right\}$. Let $\left|V\left(T^{(2)}\right)\right|=t$. We distinguish two cases.

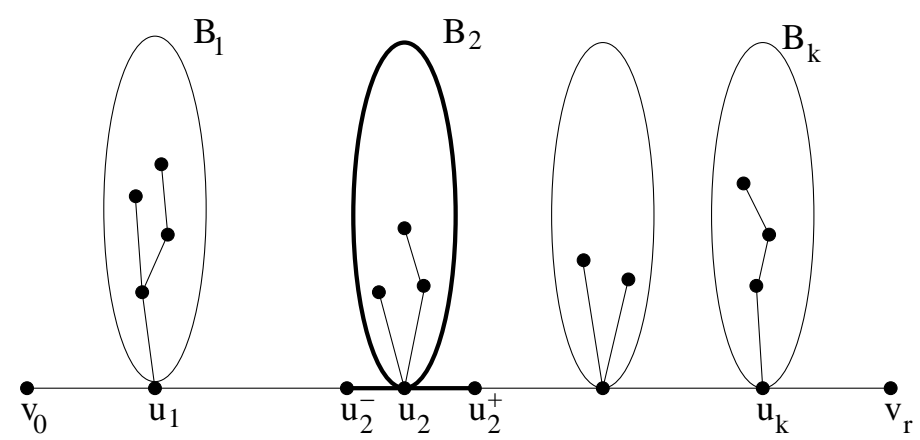

Figure 8: A path with branches. 
Subcase 1.1: $\operatorname{hom}\left(T_{m}, T^{(2)}\right)<(t-2) 2^{m-1}+2$. Consider the following trees $G_{1}$ and $G_{2} . G_{1}$ is the tree spanned by the vertices $v_{0} P u_{2}^{+}$and the branches $B_{1}, B_{2} . G_{2}$ is the tree spanned by the vertices $u_{2}^{-} P v_{r}$ and the branches $B_{2}, \ldots, B_{k}$. Note that $G_{1} \cup G_{2}=T_{n}$, $G_{1} \cap G_{2}=T^{(2)}$ and $G_{1}, G_{2}$ contains at least 4 leaves, because $k \geqslant 3$. By the hypothesis of the lemma we have

$$
\operatorname{hom}\left(T_{m}, G_{i}\right) \geqslant\left(\left|V\left(G_{i}\right)\right|-2\right) 2^{m-1}+2
$$

for $i=1,2$. Hence

$$
\begin{gathered}
\operatorname{hom}\left(T_{m}, T_{n}\right) \geqslant \operatorname{hom}\left(T_{m}, G_{1}\right)+\operatorname{hom}\left(T_{m}, G_{2}\right)-\operatorname{hom}\left(T_{m}, G_{1} \cap G_{2}\right) \geqslant \\
\geqslant\left(\left|V\left(G_{1}\right)\right|-2\right) 2^{m-1}+2+\left(\left|V\left(G_{2}\right)\right|-2\right) 2^{m-1}+2-\left(\left(\left|V\left(G_{1} \cap G_{2}\right)\right|-2\right) 2^{m-1}+2\right)= \\
=\left(\left(\left|V\left(G_{1} \cup G_{2}\right)\right|-2\right) 2^{m-1}+2=(n-2) 2^{m-1}+2 .\right.
\end{gathered}
$$

In this case we are done.

Case 1.2: $\operatorname{hom}\left(T_{m}, T^{(2)}\right) \geqslant(t-2) 2^{m-1}+2$. Consider the following trees $G_{1}$ and $G_{2}$. $G_{1}$ is the tree spanned by the vertices $\left(V\left(T_{n}\right) \backslash V\left(T^{(2)}\right)\right) \cup\left\{u_{2}^{-}, u_{2}, u_{2}^{+}\right\}$. $G_{2}$ is simply $T^{(2)}$. Note that $G_{1} \cup G_{2}=T_{n}, G_{1} \cap G_{2}=\left\{u_{2}^{-}, u_{2}, u_{2}^{+}\right\}=P_{3}$ and $G_{1}$ contains at least 4 leaves. By the hypothesis of the lemma we have

$$
\operatorname{hom}\left(T_{m}, G_{1}\right) \geqslant\left(\left|V\left(G_{1}\right)\right|-2\right) 2^{m-1}+2 .
$$

We also know that in this case

$$
\operatorname{hom}\left(T_{m}, G_{2}\right) \geqslant\left(\left|V\left(G_{2}\right)\right|-2\right) 2^{m-1}+2
$$

Note that

$$
\operatorname{hom}\left(T_{m}, P_{3}\right) \leqslant \operatorname{hom}\left(S_{m}, P_{3}\right)=2^{m-1}+2
$$

Then

$$
\begin{gathered}
\operatorname{hom}\left(T_{m}, T_{n}\right) \geqslant \operatorname{hom}\left(T_{m}, G_{1}\right)+\operatorname{hom}\left(T_{m}, G_{2}\right)-\operatorname{hom}\left(T_{m}, G_{1} \cap G_{2}\right) \geqslant \\
\geqslant\left(\left|V\left(G_{1}\right)\right|-2\right) 2^{m-1}+2+\left(\left|V\left(G_{2}\right)\right|-2\right) 2^{m-1}+2-\left(\left(\left|V\left(G_{1} \cap G_{2}\right)\right|-2\right) 2^{m-1}+2\right)= \\
=\left(\left(\left|V\left(G_{1} \cup G_{2}\right)\right|-2\right) 2^{m-1}+2=(n-2) 2^{m-1}+2 .\right.
\end{gathered}
$$

In this case we are done too.

Case 2: All maximal paths of $T_{n}$ have at most 2 branches. In the following we show that they have quite simple structure: they are starlike or double starlike trees, see Figure 9.

Let $v_{1}$ be a vertex of $T_{n}$ of degree at least 3 . Let us decompose $T_{n}$ to the branches $B_{1}^{\prime}, B_{2}^{\prime}, \ldots B_{k}^{\prime}$ at $v_{1}$. So $v_{1}$ is a leaf in the trees $B_{1}^{\prime}, B_{2}^{\prime}, \ldots B_{k}^{\prime}$. We show that all except at most one of $B_{1}^{\prime}, B_{2}^{\prime}, \ldots, B_{k}^{\prime}$ are paths. Assume that, for instance, $B_{1}^{\prime}, B_{2}^{\prime}$ are not paths. Then they contains at least two leaves of $T_{n}$ : $B_{1}^{\prime}$ contains $u_{1}, u_{2}, B_{2}^{\prime}$ contains $u_{3}, u_{4}$. Then the maximal path $u_{1} P u_{3}$ has at least three branches: one-one inside the branches $B_{1}^{\prime}$ and $B_{2}^{\prime}$ and $B_{3}^{\prime}$ at the vertex $v$. If all branches are paths, then we are done: $T_{n}$ is starlike. If 


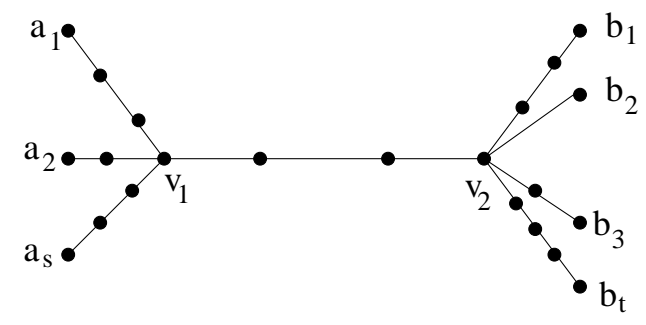

Figure 9: A double starlike tree.

one of them is not path, say $B_{1}^{\prime}$, then let us consider the vertex $v_{2} \in V\left(B_{1}^{\prime}\right)$ having degree at least 3 which is closest to $v_{1}$. Repeating the previous argument to $v_{2}$ instead of $v_{1}$, all except one branches at $v_{2}$ must be path and we also know that the branch containing $v_{1}$ is not path. Hence the tree is double starlike, where the middle path is $v_{1} P v_{2}$.

We can consider a starlike tree as a double starlike tree, where $v_{1}=v_{2}$. Let $a_{1}, \ldots, a_{s}$ and $b_{1}, \ldots, b_{t}$ be the leaves of $T_{n}$, where $a_{1}, \ldots, a_{s}$ are closer to $v_{1}$ than to $v_{2}$, while $b_{1}, \ldots, b_{t}$ are closer to $v_{2}$ than to $v_{1}$. If $v_{1}=v_{2}$ we just decompose the set of leaves into two sets of (almost) equal size. Note that $s, t \geqslant 2$. Since we can assume that there are at least 5 leaves, we assume that $s+t \geqslant 5$.

If $u_{1}, \ldots, u_{\ell}$ are some vertices of a tree, then we say that the tree spanned by $u_{1}, \ldots, u_{\ell}$ is the smallest subtree which contains the vertices $u_{1}, \ldots, u_{\ell}$. It is

$$
\operatorname{span}\left(u_{1}, \ldots, u_{\ell}\right)=\cup_{1 \leqslant i, j \leqslant \ell} u_{i} P u_{j}
$$

Subcase 2.1: If $s \geqslant 3$ and $t \geqslant 3$ both hold, then let $G_{1}$ be the tree spanned by the vertices $a_{1}, a_{2}, b_{1}, b_{2}$, and let $G_{2}$ be the tree spanned by the vertices $a_{2}, \ldots a_{s}, b_{2}, \ldots, b_{t}$. Then $G_{1} \cup G_{2}=T_{n}, G_{1} \cap G_{2}=a_{2} P b_{2}$ and both $G_{1}, G_{2}$ have at least 4 leaves. Since

$$
\begin{gathered}
\operatorname{hom}\left(T_{m}, G_{1} \cap G_{2}\right) \leqslant \operatorname{hom}\left(S_{m}, G_{1} \cap G_{2}\right)= \\
=\operatorname{hom}\left(S_{m}, a_{2} P b_{2}\right)=\left(\left|V\left(G_{1} \cap G_{2}\right)\right|-2\right) 2^{m-1}+2,
\end{gathered}
$$

we have

$$
\begin{gathered}
\operatorname{hom}\left(T_{m}, T_{n}\right) \geqslant \operatorname{hom}\left(T_{m}, G_{1}\right)+\operatorname{hom}\left(T_{m}, G_{2}\right)-\operatorname{hom}\left(T_{m}, G_{1} \cap G_{2}\right) \geqslant \\
\geqslant\left(\left|V\left(G_{1}\right)\right|-2\right) 2^{m-1}+2+\left(\left|V\left(G_{2}\right)\right|-2\right) 2^{m-1}+2-\left(\left(\left|V\left(G_{1} \cap G_{2}\right)\right|-2\right) 2^{m-1}+2\right)= \\
=\left(\left|V\left(G_{1} \cup G_{2}\right)\right|-2\right) 2^{m-1}+2=(n-2) 2^{m-1}+2 .
\end{gathered}
$$

Hence we are done in this case.

Subcase 2.2: If $s \geqslant 4$ and $t=2$ then let $G_{1}$ be the tree spanned by $a_{1}, a_{2}, b_{1}, b_{2}$ and let $G_{2}$ be the tree spanned by $a_{2}, \ldots, a_{k}, b_{1}$. Then $G_{1} \cup G_{2}=T_{n}, G_{1} \cap G_{2}=a_{2} P b_{1}$ and both $G_{1}, G_{2}$ have at least 4 leaves. In this case we are done as before. Clearly, the case $s \geqslant 2$ and $t \geqslant 4$ is completely similar. 
Subcase 2.3: The last case is $s=3, t=2$ (and $s=2, t=3$ ). Let $G_{1}=$ $\operatorname{span}\left(a_{1}, a_{2}, b_{1}, b_{2}\right), G_{2}=\operatorname{span}\left(a_{2}, a_{3}, b_{1}, b_{2}\right), G_{3}=\operatorname{span}\left(a_{2}, b_{1}, b_{2}\right), G_{4}=\left(a_{1}, a_{2}, a_{3}, v_{2}\right)$. Then $G_{1} \cap G_{2}=G_{3}, G_{3} \cap G_{4}=a_{2} P v_{2}$. Note that $G_{1}, G_{2}, G_{4}$ has 4 leaves, thus

$$
\operatorname{hom}\left(T_{m}, G_{i}\right) \geqslant\left(\left|V\left(G_{i}\right)\right|-2\right) 2^{m-1}+2
$$

for $i=1,2,4$. If $\operatorname{hom}\left(T_{m}, G_{3}\right) \leqslant\left(\left|V\left(G_{3}\right)\right|-2\right) 2^{m-1}+2$, then from $T_{n}=G_{1} \cup G_{2}, G_{3}=G_{1} \cap$ $G_{2}$ we obtain that hom $\left(T_{m}, T_{n}\right) \geqslant(n-2) 2^{m-1}+2$. If hom $\left(T_{m}, G_{3}\right) \geqslant\left(\left|V\left(G_{3}\right)\right|-2\right) 2^{m-1}+2$, then from $T_{n}=G_{3} \cap G_{4}$ we obtain that $\operatorname{hom}\left(T_{m}, T_{n}\right) \geqslant(n-2) 2^{m-1}+2$. Hence we are done in this case as well.

Proof of Theorem 5.2. The result immediately follows from Lemma 5.4 and Proposition 5.5 below.

Proposition 5.5. Let $T_{n}$ be a tree on $n$ vertices with exactly four leaves. Then for any tree $T_{m}$ on $m$ vertices we have

$$
\operatorname{hom}\left(T_{m}, T_{n}\right) \geqslant(n-2) 2^{m-1}+2,
$$

where $n$ is the number of vertices of $T_{n}$.

Proof. If $T_{n}$ has a vertex of degree 4 then by Theorem 4.6 we have

$$
\operatorname{hom}\left(T_{m}, T_{n}\right) \geqslant 2(n-1) C^{m-2},
$$

where

$$
C=\left(\prod_{i=1}^{n} d_{i}^{d_{i}}\right)^{1 / 2 e\left(T_{n}\right)}=2
$$

Hence

$$
\operatorname{hom}\left(T_{m}, T_{n}\right) \geqslant(n-1) 2^{m-1} \geqslant(n-2) 2^{m-1}+2 .
$$

For the case when $T_{n}$ has two vertices of degree 3 , we need more preparation.

Lemma 5.6. Let $T_{n}$ be a tree with exactly 4 leaves and two vertices of degree 3 . Let $x$ and $y$ be the vertices of $T_{n}$ with degree 3 . Assume that there are at most 3 vertices of $T_{n}$ which have degree 2 and not on the path $x P y$. Then for any tree $T_{m}$ on $m$ vertices we have

$$
\operatorname{hom}\left(T_{m}, T_{n}\right) \geqslant(n-2) 2^{m-1}+2,
$$

where $n$ is the number of vertices of $T_{n}$.

Proof. We can assume that $m \geqslant 4$, otherwise the statement is trivial. We prove the slightly stronger inequality

$$
\operatorname{hom}\left(T_{m}, T_{n}\right)>\left(n-2+\frac{1}{8}\right) 2^{m-1} .
$$


If $m \geqslant 4$, then this implies that

$$
\operatorname{hom}\left(T_{m}, T_{n}\right)>(n-2) 2^{m-1}+1
$$

or equivalently,

$$
\operatorname{hom}\left(T_{m}, T_{n}\right) \geqslant(n-2) 2^{m-1}+2 .
$$

To prove this statement we use Theorem 4.1 with a suitable Markov chain. Let $p_{i j}=\frac{1}{2}$ for $(i, j) \in E\left(T_{n}\right)$ if $i$ has degree 2. Naturally, $p_{i j}=1$ if $(i, j) \in E(G)$ and $i$ is a leaf. Finally, if $i \in\{x, y\}, j \in x P y$ then $p_{i j}=\frac{1}{2}$ and if $i \in\{x, y\}, j \notin x P y$ then $p_{i j}=\frac{1}{4}$.

Let $r$ be the number of vertices of $x P y$. Then $n=r+t+4$ for an integer $t \geqslant 0$. Let $N=4 r+2 t+4$. Then the stationary distribution is the following: $q_{i}=\frac{4}{N}$ if $i \in x P y$, $q_{i}=\frac{2}{N}$ if $i \notin x P y$, but has degree 2 and finally, $q_{i}=\frac{1}{N}$ if $i$ is a leaf.

Then

$$
H(P \mid Q)=\frac{N-12}{N} \log 2+\frac{8}{N}\left(\frac{1}{2} \log 2+\frac{1}{2} \log 4\right)=\log 2 .
$$

On the other hand,

$$
\begin{aligned}
& H(Q)+2(H(D \mid Q)-H(P \mid Q)) \\
= & \left(\frac{4 r}{N} \log \frac{N}{4}+\frac{2 t}{N} \log \frac{N}{2}+\frac{4}{N} \log \frac{N}{1}\right)+2 \frac{8}{N}\left(\log 3-\frac{3}{2} \log 2\right) \\
= & \log \frac{N}{4}+\frac{2 t}{N} \log 2+\frac{16(\log 3-\log 2)}{N} .
\end{aligned}
$$

Note that

$$
\begin{aligned}
\log \left(n-2+\frac{1}{8}\right)-\log \frac{N}{4} & \leqslant \int_{N / 4}^{n-2} \frac{1}{x} d x \\
& \leqslant \frac{n-2+\frac{1}{8}-N / 4}{N / 4} \\
& =\frac{4}{N}\left(\frac{t}{2}+1+\frac{1}{8}\right) \\
& =\frac{1}{N}\left(2 t+\frac{9}{2}\right) .
\end{aligned}
$$

Hence if

$$
\frac{1}{N}\left(2 t+\frac{9}{2}\right) \leqslant \frac{2 t}{N} \log 2+\frac{16(\log 3-\log 2)}{N}
$$

then

$$
\log \left(n-2+\frac{1}{8}\right) \leqslant H(Q)+2(H(D \mid Q)-H(P \mid Q))
$$

consequently

$$
\operatorname{hom}\left(T_{k}, G\right) \geqslant \exp (H(Q)+2 H(D \mid Q)+(m-3) H(P \mid Q))>\left(n-2+\frac{1}{8}\right) 2^{m-1} .
$$


The above inequality is satisfied if

$$
t \leqslant \frac{8 \log \frac{3}{2}-\frac{9}{4}}{1-\log 2} \approx 3.238
$$

This proves the statement of the theorem.

Lemma 5.7. Let $T_{n}$ be a tree obtained from a path on $n-8$ vertices by gluing one-one $P_{5}$ at the middle vertices to both ends of the path $P_{n-8}$ (see Fig. 10). Then for any tree $T_{m}$ on $m$ vertices we have

$$
\operatorname{hom}\left(T_{m}, T_{n}\right) \geqslant(n-1) 2^{m-1} .
$$

Proof. We will show by induction on $m$ that

$$
\operatorname{hom}\left(T_{m}, T_{n}\right) \geqslant(n-1) 2^{m-1} .
$$

Let $v$ be any leaf of $T_{m}$ with unique neighbor $u$ and let $T_{m-1}=T_{m}-v$ be a rooted tree with root $u$.

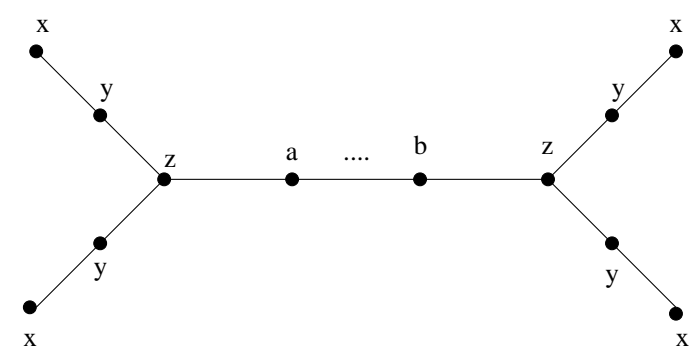

Figure 10: Special double starlike trees.

Let us use the hom-vectors of the Fig. 10, that is

$$
\mathbf{h}\left(T_{m-1}, u, T_{n}\right)=(x, x, y, y, z, a, \ldots, b, z, y, y, x, x) .
$$

Now suppose that

$$
\operatorname{hom}\left(T_{m-1}, T_{n}\right) \geqslant(n-1) 2^{m-2} .
$$

It is easy to see by induction that $z>2 x$ if $T_{m-1}$ has at least two vertices. By tree-walk algorithm, we have

$$
\begin{aligned}
\operatorname{hom}\left(T_{m}(v), T_{n}\right) & =4 x+8 y+6 z+2(a+\cdots+b) \\
& \geqslant 8 x+8 y+4 z+2(a+\cdots+b) \\
& =2 \operatorname{hom}\left(T_{m-1}, G\right) \\
& \geqslant(n-1) 2^{m-1},
\end{aligned}
$$

which shows (5.3). 

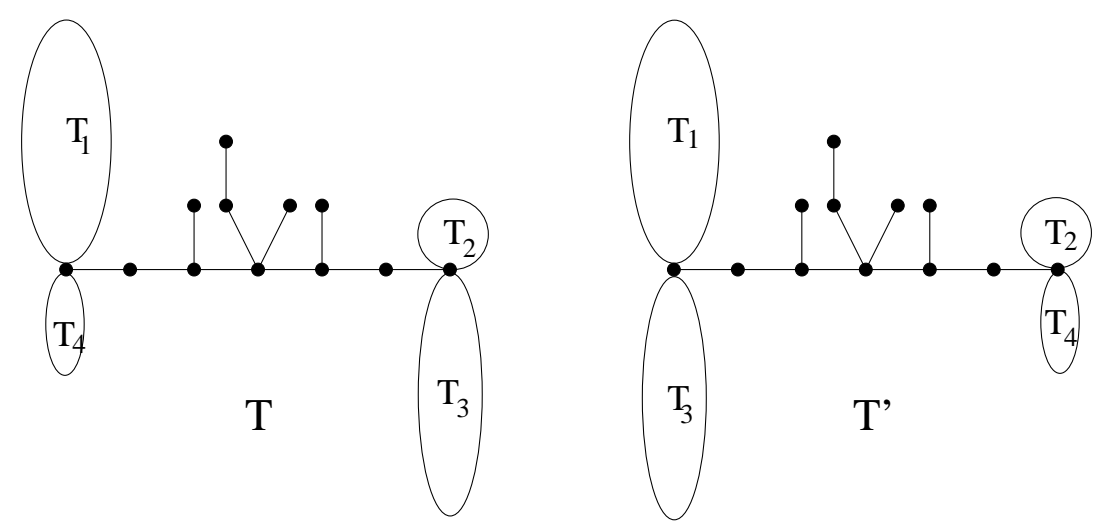

Figure 11: LS-switch.

Next we introduce a transformation which we will call LS-switch (Large-Small switch).

Definition 5.8 (LS-switch). Let $R(u, v)$ be a tree with specified vertices $u$ and $v$ such that the distance of $u$ and $v$ is even and $R$ has an automorphism of order 2 which exchanges the vertices $u$ and $v$. Let $T_{1}(x), T_{2}(x), T_{3}(y), T_{4}(y)$ be rooted trees such that $T_{2}(x)$ is the rooted subtree of $T_{1}(x)$ and $T_{4}(y)$ is the rooted subtree of $T_{3}(y)$. Let the tree $T$ be obtained from the trees $R(u, v), T_{1}(x), T_{2}(x), T_{3}(y), T_{4}(y)$ by attaching a copy of $T_{1}(x), T_{4}(y)$ to $R(u, v)$ at vertex $u$ and a copy of $T_{2}(x), T_{3}(y)$ at vertex $v$. Assume that the tree $T^{\prime}$ is obtained from the trees $R(u, v), T_{1}(x), T_{2}(x), T_{3}(y), T_{4}(y)$ by attaching a copy of $T_{1}(x), T_{3}(y)$ to $R(u, v)$ at vertex $u$ and a copy of $T_{2}(x), T_{4}(y)$ at vertex $v$. Then $T^{\prime}$ is the LS-switch of $T$. Observe that there is a natural bijection between the color classes of $T^{\prime}$ and $T$.

A particular case of the LS-switch is when $R(u, v)$ is a path of even length with end vertices $u$ and $v, T_{2}(x)$ and $T_{4}(y)$ are one-vertex rooted trees, then $T^{\prime}$ is obtained from $T$ by an even-KC-transformation, i.e., KC-transformation according to a path of even length. Another useful special case is when $R(u, v)$ is a tree where we attach an arbitrary tree to the middle vertex of the path on 3 vertices and $u$ and $v$ are the end vertices of the path (in this case the automorphism simply switches $u$ and $v$ ), and $T_{2}(x), T_{4}(y)$ are the rooted trees with 1 vertex, in this case we get back to a particular case of the original Kelmans-transformation [12].

The following theorem with respect to the LS-switch is just an extension of the even case of Theorem 1.3.

Theorem 5.9. Let $T^{\prime}$ be the LS-switch of $T$. Let $H$ be an arbitrary tree. Then

$$
\operatorname{hom}(H, T) \leqslant \operatorname{hom}\left(H, T^{\prime}\right)
$$

Proof. (Sketch.) The unique shortest path connecting all $T_{i}$ 's in $T$ (or $T^{\prime}$ ) will be denoted by $P_{2 k}$, a path of even length with vertices labeled consecutively by $0,1, \ldots, 2 k$. Without loss of generality, we can assume that $0 \in V\left(T_{1}\right)$. For $1 \leqslant j \leqslant 2 k-1$, let $A_{j}$ denote the component of $T$ that contains the vertex $j$ when we delete all edges of $P_{2 k}$. By the definition of LS-switch, the subtrees $A_{j}$ and $A_{2 k-j}$ are isomorphic, so we can identify 
$V\left(A_{j}\right) \backslash\{j\}$ with $V\left(A_{2 k-j}\right) \backslash\{2 k-j\}$. We will also consider $V\left(T_{2}\right) \backslash\{0,2 k\}$ as the subset of $V\left(T_{1}\right) \backslash\{0,2 k\}$ and $V\left(T_{4}\right) \backslash\{0,2 k\}$ as the subset of $V\left(T_{3}\right) \backslash\{0,2 k\}$.

Let $v$ be a vertex of $H$. For $0 \leqslant s \leqslant 2 k, u \in V\left(T_{i}\right) \backslash\{0,2 k\}(1 \leqslant i \leqslant 4)$ and $a \in V\left(A_{j}\right) \backslash\{j\}(1 \leqslant j \leqslant 2 k-1)$, we define

$$
\begin{gathered}
p_{s}:=\mid\left\{\{ f \in \operatorname { H o m } ( H , T ) : f ( v ) = m \} | , p _ { s } ^ { \prime } : = | \left\{\left\{f \in \operatorname{Hom}\left(H, T^{\prime}\right): f(v)=m\right\} \mid,\right.\right. \\
t_{i}(u):=|\{f \in \operatorname{Hom}(H, T): f(v)=u\}|, t_{i}^{\prime}(u):=\left|\left\{f \in \operatorname{Hom}\left(H, T^{\prime}\right): f(v)=u\right\}\right|,
\end{gathered}
$$

and

$$
p_{j}(a):=|\{f \in \operatorname{Hom}(H, T): f(v)=a\}|, p_{j}^{\prime}(a):=\left|\left\{f \in \operatorname{Hom}\left(H, T^{\prime}\right): f(v)=a\right\}\right| .
$$

We prove by induction that the following inequalities are preserved by the steps of the tree-walk algorithm. For any $0 \leqslant s \leqslant k, a \in V\left(A_{j}\right) \backslash\{j\}(1 \leqslant j \leqslant k), u \in V\left(T_{2}\right) \backslash\{0,2 k\}$, $w \in V\left(T_{4}\right) \backslash\{0,2 k\}, x \in V\left(T_{1}\right) \backslash V\left(T_{2}\right)$ and $y \in V\left(T_{3}\right) \backslash V\left(T_{4}\right)$ we have

$$
\begin{aligned}
p_{k-s}^{\prime}+p_{k+s}^{\prime} & \geqslant p_{k-s}+p_{k+s} \text { and } p_{k-s}^{\prime} \geqslant p_{k+s}, p_{k-s} \\
p_{j}^{\prime}(a)+p_{2 k-j}^{\prime}(a) & \geqslant p_{j}(a)+p_{2 k-j}(a) \text { and } p_{j}^{\prime}(a) \geqslant p_{2 k-j}(a), p_{j}(a) \\
t_{1}^{\prime}(u)+t_{2}^{\prime}(u) & \geqslant t_{1}(u)+t_{2}(u) \text { and } t_{1}^{\prime}(u) \geqslant t_{1}(u), t_{2}(u) \\
t_{3}^{\prime}(w)+t_{4}^{\prime}(w) & \geqslant t_{3}(w)+t_{4}(w) \text { and } t_{3}^{\prime}(w) \geqslant t_{3}(w), t_{4}(w) \\
t_{1}^{\prime}(x) & \geqslant t_{1}(x) \text { and } t_{3}^{\prime}(y) \geqslant t_{3}(y) .
\end{aligned}
$$

We only need to check that the two operations in the tree-walk algorithm preserve all the above inequalities, which is routine and left to the reader.

Now assume that $T_{n}$ has two vertices, $x$ and $y$, of degree 3. Among these trees $(n$ vertices, 4 leaves, two vertices of degree 3 ) let us choose $\overline{T_{n}}$ to be the one for which $\operatorname{hom}\left(T_{m}, \overline{T_{n}}\right)$ is minimal and among these trees the length of the path is maximal.

Let the four leaves of $\overline{T_{n}}$ denoted by $z_{1}, z_{2}, z_{3}, z_{4}$ such that $z_{1}, z_{2}$ are closer to $x$ than $y$, and $z_{3}, z_{4}$ are closer to $y$ than $x$. Let the number of edges of $x P y, x P z_{1}, x P z_{2}, y P z_{3}, y P z_{4}$ be $a, b, c, d, e$, respectively. We show that $\max (b, c, d, e) \leqslant 2$. Indeed, if say $b>2$ then $\overline{T_{n}}$ can be obtained by an LS-switch from a graph $T_{n}^{*}$ as follows.

If $b$ is even, then let $u$ be the unique vertex such that $d\left(z_{1}, u\right)=2$. Then $u P x=R(u, x)$ is a path of even length. Let $T_{2}=z_{1} P u$. Furthermore, let $T_{1}$ be the tree spanned by the vertices $x, z_{3}, z_{4} . T_{3}=x P z_{2}$ and $T_{4}=\{u\}$. Then $T_{2}$ is a rooted subtree of $T_{1}$ and $T_{4}$ is a rooted subtree of $T_{3}$. Now making an inverse LS-swith we obtain $T_{n}^{*}$. By Theorem 5.9, we know that

$$
\operatorname{hom}\left(T_{m}, \overline{T_{n}}\right) \geqslant \operatorname{hom}\left(T_{m}, T_{n}^{*}\right)
$$

and in $T_{n}^{*}$, the vertices of degree $3, y$ and $u$, has distance $a+b-2>a$ contradicting the choice of $\overline{T_{n}}$.

If $b$ is odd, then let $u$ be the unique neighbor of $z_{1}$ and we repeat the previous argument. The distance of $u$ and $x$ is even again. 
Hence we can assume that $\max (b, c, d, e) \leqslant 2$. If not all of them are 2 , then we can use Lemma 5.6 to get that

$$
\operatorname{hom}\left(T_{m}, \overline{T_{n}}\right) \geqslant(n-2) 2^{m-1}+2 .
$$

If $b=c=d=e=2$, then we use Lemma 5.7 to obtain that

$$
\operatorname{hom}\left(T_{m}, \overline{T_{n}}\right) \geqslant(n-1) 2^{m-1} \geqslant(n-2) 2^{m-1}+2 .
$$

This completes the proof of Proposition 5.5.

\subsection{Trees with 3 leaves}

Lemma 5.10. (a) Let $n=a+b+c+1$, and $\min (a, b, c) \geqslant 2$. Then for any tree $T_{m}$ on $m$ vertices we have

$$
\operatorname{hom}\left(T_{m}, Y_{a, b, c}\right) \geqslant(n-2) 2^{m-1}+2 \text {. }
$$

(b) Let $n=a+b+2$, and $\min (a, b) \geqslant 3$. Then for any tree $T_{m}$ on $m$ vertices we have

$$
\operatorname{hom}\left(T_{m}, Y_{a, b, 1}\right) \geqslant(n-2) 2^{m-1}+2 \text {. }
$$

Proof. (a)

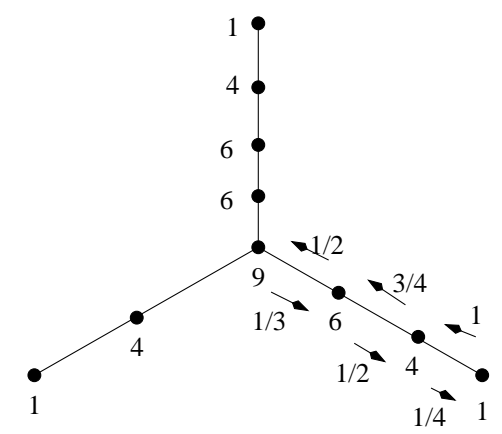

Figure 12: $Y_{a, b, c}$ where $\min (a, b, c) \geqslant 2$ with a special Markov chain.

We can think to $Y_{a, b, c}$ with $\min (a, b, c) \geqslant 2$ as follows: we consider $Y_{2,2,2}$ and we subdivide the edges between the vertex of degree 3 and its neighbors a few times. Let us write the weights $1,4,1,4,1,4,9$ to the vertices of $Y_{2,2,2}$ according to the figure and then let us write weights 6 on the new vertices obtained by subdivision. It is easy to check that there is a unique Markov chain on $Y_{a, b, c}$, where the stationary distribution is proportional to the weights. (In fact, we write a few transition probabilities on the figure.)

It is easy to check that $H(P \mid Q)=\log 2$ and if $N=24+6(n-7)=6(n-3)$, then

$$
H(Q)+2(H(D \mid Q)-H(P \mid Q))
$$




$$
\begin{aligned}
= & \left(\frac{9}{N} \log \frac{N}{9}+\frac{12}{N} \log \frac{N}{4}+\frac{3}{N} \log \frac{N}{1}+\frac{6(n-7)}{N} \log \frac{N}{6}\right)+ \\
& +2 \cdot \frac{12}{N}\left(\log 2-\left(\frac{1}{4} \log 4+\frac{3}{4} \log \frac{4}{3}\right)\right) \\
= & \log \frac{N}{6}+\frac{9}{N} \log \frac{6}{9}+\frac{12}{N} \log \frac{6}{4}+\frac{3}{N} \log \frac{6}{1}+\frac{24}{N}\left(\log 2-\left(\frac{1}{4} \log 4+\frac{3}{4} \log \frac{4}{3}\right)\right) \\
= & \log (n-3)+\frac{24}{N} \log \frac{3}{2} .
\end{aligned}
$$

Since

$$
\log (n-2+\varepsilon)-\log (n-3)=\int_{n-3}^{n-2+\varepsilon} \frac{d x}{x} \leqslant \frac{1+\varepsilon}{n-3}=\frac{6}{N}(1+\varepsilon)
$$

we can choose $\varepsilon=4 \log \frac{3}{2}-1>\frac{1}{2}$ to deduce that

$$
\operatorname{hom}\left(T_{m}, Y_{a, b, c}\right) \geqslant(n-2+\varepsilon) 2^{m-1} .
$$

This is already greater than $(n-2) 2^{m-1}+2$ for $m \geqslant 3$. The statement is trivial for $m \leqslant 2$.

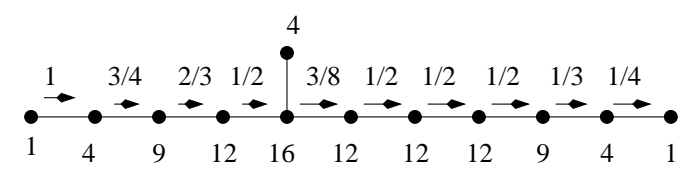

Figure 13: $Y_{a, b, 1}$ where $\min (a, b) \geqslant 3$ with a special Markov chain.

We use completely the same argument as in part (a). We think to $Y_{a, b, 1}$ as a subdivision of $Y_{3,3,1}$ and use the Markov chain on the figure. Again we have $H(P \mid Q)=\log 2$ and the sum of the weights is $N=48+12(n-8)=12(n-4)$. Hence

$$
H(Q)+2(H(D \mid Q)-H(P \mid Q))=\log \frac{N}{12}+\frac{12}{N}\left(\frac{11}{3} \log 3-\frac{1}{3} \log 2\right) .
$$

Since

$$
\log (n-2+\varepsilon)-\log (n-4)=\int_{n-4}^{n-2+\varepsilon} \frac{d x}{x} \leqslant \frac{2+\varepsilon}{n-4}=\frac{12}{N}(2+\varepsilon)
$$

we can choose $\varepsilon=\frac{11}{3} \log 3-\frac{1}{3} \log 2-2>1.79$ to deduce that

$$
\operatorname{hom}\left(T_{m}, Y_{a, b, c}\right) \geqslant(n-2+\varepsilon) 2^{m-1} .
$$

This is already greater than $(n-2) 2^{m-1}+2$ for $m \geqslant 2$. The statement is trivial for $m=1$. 
Remark 5.11. Since every Markov chains is reversible on a tree, there is a natural way to define a new Markov chain on a subdivided edge. Assume that the probabilities of the stationary distribution were $q_{i}, q_{j}$ and $p_{i j}, p_{j i}$ were the transition probabilities at the vertices $i, j$. Then $q_{i} p_{i j}=q_{j} p_{j i}$ (reversibility) and we can put a vertex $r$ with weight $2 q_{i} p_{i j}$ and $p_{r i}=p_{r j}=1 / 2$ on the edge $(i, j)$. Then the new stationary distribution will be proportional to the weights $\left\{q_{i} \mid i \in V(T)\right\} \cup\left\{2 q_{i} p_{i j}\right\}$.

Theorem 1.6. Let $T_{n}$ be a tree on $n$ vertices. Assume that for a tree $T_{m}$ we have

$$
\operatorname{hom}\left(T_{m}, T_{n}\right)<\operatorname{hom}\left(T_{m}, P_{n}\right) .
$$

Then $T_{n}=Y_{1,1, n-3}$ and $n$ is even.

Proof. Note that if $T_{n}$ has at least 4 leaves then Theorem 5.5 implies that

$$
\operatorname{hom}\left(T_{m}, T_{n}\right) \geqslant(n-2) 2^{m-1}+2=\operatorname{hom}\left(S_{m}, P_{n}\right) \geqslant \operatorname{hom}\left(T_{m}, P_{n}\right)
$$

contradicting to the condition of the theorem. Hence $T_{n}=Y_{a, b, c}$ for some $a, b, c$. Observe that if one of $a, b, c$ is even then $Y_{a, b, c}$ can be obtained from $P_{n}$ by an even-KCtransformation and then Theorem 1.3 implies

$$
\operatorname{hom}\left(T_{m}, T_{n}\right) \geqslant \operatorname{hom}\left(T_{m}, P_{n}\right)
$$

contradicting to the condition of the theorem. Note that if $n$ is odd, then one of $a, b, c$ is necessarily even and so we are done. From Lemma 5.10 we also know that $\min (a, b, c)=1$, say $c=1$ and $\min (a, b) \leqslant 2$. But then $\min (a, b)=1$, because it must be odd. Hence $T_{n}=Y_{1,1, n-3}$ and $n$ is even.

Remark 5.12. There is a tree $T_{m}$ for which hom $\left(T_{m}, S_{4}\right)<\operatorname{hom}\left(T_{m}, P_{4}\right)$. On Fig. 14 one

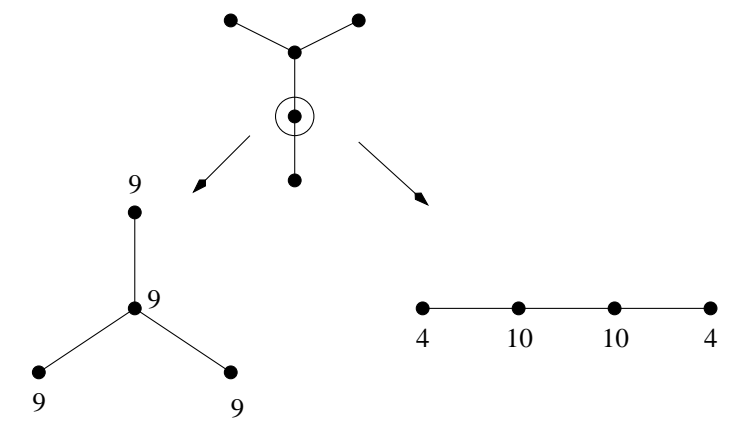

Figure 14: An example.

can see a rooted tree and its homomorphism vectors to $S_{4}$ and $P_{4}$. Now if we attach $k$ copies of this rooted tree at the root then for the obtained tree $T_{m}$ we have

$$
\operatorname{hom}\left(T_{m}, P_{4}\right)=2 \cdot 4^{k}+2 \cdot 10^{k}>4 \cdot 9^{k}=\operatorname{hom}\left(T_{m}, S_{4}\right)
$$

for large enough $k$.

On the other hand, it seems that $\operatorname{hom}\left(T_{m}, Y_{1,1, n-3}\right) \geqslant \operatorname{hom}\left(T_{m}, P_{n}\right)$ if $n \geqslant 6$ and even. 


\section{Open problems}

We collected a few open problems and conjectures in this section.

We first recall a conjecture from the Introduction, namely that there is no exceptional case in Theorem 1.6 if $n \geqslant 5$.

Conjecture 1.7 Let $T_{n}$ be a tree on $n$ vertices, where $n \geqslant 5$. Then for any tree $T_{m}$ we have

$$
\operatorname{hom}\left(T_{m}, P_{n}\right) \leqslant \operatorname{hom}\left(T_{m}, T_{n}\right)
$$

Note that to prove Conjecture 1.7, one only needs to prove that for any tree $T_{m}$ we have

$$
\operatorname{hom}\left(T_{m}, P_{n}\right) \leqslant \operatorname{hom}\left(T_{m}, Y_{1,1, n-3}\right)
$$

for $n \geqslant 6$, where $n$ is even.

There is also an open problem in Figure 4, if true, would provide an alternative proof of the first part of Theorem 1.8 (through Theorem 1.2).

Problem 6.1. Is it true that

$$
\operatorname{hom}\left(P_{n}, T_{n}\right) \leqslant \operatorname{hom}\left(T_{n}, T_{n}\right)
$$

for every tree $T_{n}$ on $n$ vertices?

We believe that the answer is affirmative for this question. This question naturally leads to the following problem.

Problem 6.2. Characterize all graphs $G$ for which

$$
\operatorname{hom}\left(P_{m}, G\right) \leqslant \operatorname{hom}\left(T_{m}, G\right)
$$

for all $m$ and all trees $T_{m}$ on $m$ vertices.

Note that if $G$ is $d$-regular, then $\operatorname{hom}\left(P_{m}, G\right)=\operatorname{hom}\left(T_{m}, G\right)=|V(G)| d^{m-1}$. We have also seen that the inequality of Problem 6.2 is satisfied if $G=P_{n}$ or $S_{n}$. Probably, it is hard to characterize these graphs. Maybe, it is easier to describe those graphs $G$ for which the inequality of Problem 6.2 is satisfied for large enough $m$.

The dual of Problem 6.2 is also natural:

Problem 6.3. Characterize all trees $T_{m}$ on $m$ vertices for which

$$
\operatorname{hom}\left(P_{m}, G\right) \leqslant \operatorname{hom}\left(T_{m}, G\right)
$$

for all graph $G$.

Probably, this is an easier problem than Problem 6.2. Note that already Sidorenko [19] achieved nice results on this problem. Still the problem is far from being solved.

In light of the tree-walk algorithm, it would be interesting to develop an algorithm for computing the number of homomorphisms from bipartite graphs to any graph. 


\section{Acknowledgments}

The first author is very grateful to László Lovász and Miklós Simonovits for suggesting to study the papers of Alexander Sidorenko. He also thanks Benjamin Rossman for the comments on the paper [21]. He is also very grateful to Balázs Gerencsér for the useful discussion on Markov chains and entropies.

This research was partly done while the second author was visiting the Alfréd Rényi Institute of Mathematics. He is very grateful to Miklós Abért and the Institute for their hospitality and support. He also would like to thank Yanfeng Luo for introducing the graph homomorphisms and Jiang Zeng for all his encouragement during this work.

Special thanks goes to Masao Ishikawa for suggesting the name tree-walk algorithm.

\section{References}

[1] Sr. Arworn and P. Wojtylak, An algorithm for the number of path homomorphisms, Discrete Math., 309 (2009), 5569-5573.

[2] B. Bollobás, Modern graph theory, Graduate Texts in Mathematics, 184. SpringerVerlag, New York, 1998.

[3] B. Bollobás and M. Tyomkyn, Walks and paths in trees, J. Graph Theory, 70 (2012), $54-66$.

[4] J.A. Bondy and U.S.R. Murty, Graph Theory, Springer-Verlag, New York, 2008.

[5] T.M. Cover and J.A. Thomas, Elements of information theory, 2nd ed., John Wiley \& Sons, New Jersey, 2006.

[6] P. Csikvári, On a poset of trees, Combinatorica, 30 (2010), 125-137.

[7] P. Csikvári, On a poset of trees II, J. Graph Theory, 74 (2013), 81-103.

[8] P. Csikvári and Z. Lin, Graph homomorphisms between trees, II: Path coloring trees, preprint, 2014.

[9] D. Dellamonica Jr., P. Hacell, T. Łuczak, D. Mubayi, B. Nagle, Y. Person, V. Rödl, and M. Schacht, Tree-minimal graphs are almost regular, J. Comb., 3 (2012), 49-62.

[10] M.A. Fiol and E. Garriga, Number of walks and degree powers in a graph, Discrete Math., 309 (2009), 2613-2614.

[11] C. Jagger, P. Stovicek and A. Thomason, Multiplicities of subgraphs, Combinatorica, 16 (1996), 123-141.

[12] A.K. Kelmans, On graphs with randomly deleted edges, Acta. Math. Acad. Sci. Hung., 37 (1981), 77-88.

[13] S. Kopparty and B. Rossman, The homomorphism domination exponent, European J. Combin., 32 (2011), 1097-1114.

[14] A.M. Leontovich, The number of mappings of graphs, the ordering of graphs and the Muirhead theorem, Problemy Peredachi Informatsii 25 (1989), no. 2, 91-104; translation in Problems Inform. Transmission 25 (1989) 154-165. 
[15] J.L.X. Li and B. Szegedy, On the logarithmic calculus and Sidorenko's conjecture, Combinatorica, to appear.

[16] Z. Lin and J. Zeng, On the number of congruence classes of paths, Discrete Math., 312 (2012), 1300-1307.

[17] L. Lovász and J. Pelikán, On the eigenvalues of trees, Per. Mathematica Hungarica, 3 (1973), 175-182.

[18] A. Sidorenko, A correlation inequality for bipartite graph, Graph Combin., 9 (1993), 201-204.

[19] A. Sidorenko, A partially ordered set of functionals corresponding to graphs, Discrete Math., 131 (1994), 263-277.

[20] R.P. Stanley, Enumerative combinatorics, Vol. 1, Cambridge University Press, Cambridge, 1997.

[21] B. Rossman and E. Vee, Counting homomorphisms from trees and cycles, 2006, unpublished manuscript. 